\title{
Supersymmetric construction of self-consistent condensates in the large $N$ Gross-Neveu model: Solitons on finite-gap potentials
}

\author{
Adrián Arancibia* \\ Instituto de Matemática y Física, Universidad de Talca, Casilla 747, Talca, Chile
}

(Received 2 January 2018; published 24 September 2018)

\begin{abstract}
In the present work, the set of stationary solutions of the Gross-Neveu model in the 't Hooft limit is extended. Such an extension is obtained by striving for a hidden supersymmetry associated with disconnected sets of stationary solutions. How the supersymmetry arises from the Darboux-Miura transformations between Lax pairs of the stationary modified Korteweg-de Vries and the stationary Korteweg-de Vries hierarchies is shown, associating the correspondent superpotentials with self-consistent condensates for the Gross-Neveu model.
\end{abstract}

DOI: $10.1103 /$ PhysRevD.98.065013

\section{INTRODUCTION}

The Gross-Neveu (GN) model [1] corresponds to a quantum field theory for nonlinear interacting fermions without mass. The model presents some interesting properties: dynamical mass generation, asymptotic freedom, and spontaneous breaking of symmetry. Models with interacting fermions and self-consistent condensates have been used to describe a large variety of phenomena related to soliton physics, kinks, and breathers, especially in particle physics [2,3], superconductivity [4-6], and conducting polymer models [7-9], among other areas [10,11].

In the past 40 years, nonperturbative solution methods for this field theory in the 't Hooft limit (or large $N$ limit) have been studied in detail. The first analytical solutions in this direction were obtained by applying the inverse scattering method $[12,13]$, which allowed one to relate fermionic condensates to superpotentials of pairs of reflectionless systems of the Schrödinger type in $1+1$ dimension $(1+1 \mathrm{D})$, thus bringing to light a hidden nonlinear $N=4$ supersymmetry in the stationary sector of the GN model [14-16]. The inverse scattering method has allowed the generation of an infinite family of semiclassical condensates, corresponding to one-gap (massive) Dirac potentials with solitary defects in its spectrum [17].

A more general method to construct analytical solutions was found by applying the series expansion of the Gorkov resolvent [10], obtaining as a more general condition that the GN model semiclassical stationary condensates must

*adaran.phi@gmail.com

Published by the American Physical Society under the terms of the Creative Commons Attribution 4.0 International license. Further distribution of this work must maintain attribution to the author(s) and the published article's title, journal citation, and DOI. Funded by SCOAP ${ }^{3}$. be solutions of the inhomogeneous stationary modified Korteweg-de Vries hierarchy (s-mKdVh). This led to the construction of exact periodic inhomogeneous condensate solutions known as kink crystals and kink-antikink crystals, which have already been found as self-consistent condensates through the Hartree-Fock approximation [18-20]. Such condensates correspond to two- and three-gap potentials for the Dirac Hamiltonian or Bogoliubov-de Gennes operator in $1+1 \mathrm{D}$.

The most important results in this paper are the following:

(i) The extension of the stationary scalar condensates for the Gross-Neveu model to the most general form, as finite-gap Dirac potentials with solitonic defects. Such an extension is achieved by using the supersymmetry hidden in the system. The supersymmetric method presented below allows one to evade the inverse scattering approach and to algebraically construct infinite families of extended Schrödinger Hamiltonians with central charge and nonlinear $N=4$ supersymmetry starting from the exactly solvable finite-gap Schrödinger systems. When one pair of fermionic integrals are of order one, it is possible to identify any of such fermionic integrals and the central element of the respective superalgebra with the Lax pair formulation of an equation in the s-mKdVh. Thus, the stationary condensate solutions of the GN model are identified with the superpotentials that define the set of fermionic integrals of order one. The nonlinear $N=4$ hidden supersymmetry is observed by using the Darboux-Miura transformations and the Lax pair formalism of the s-KdVh and the s-mKdVh.

(ii) From the recurrence relations in the construction of the s-mKdVh and the algebro-geometric formalism, the self-consistency equations that fix the occupation 
of the allowed states by the fermions of different flavors for each condensate are written in the form of a consistent system of equations with more unknowns than equations; therefore, there are an infinite number of solutions. As an example, a special case of ground state that allows the decoupling of the occupations among bound states is studied. For this example the occupation of each bound state depends only on its energy and the occupation of the states in the spectrum of the finitegap background.

This work is structured as follows: in Sec. II, by means of the method of series expansion of the Gorkov resolvent, the self-consistent condensates of the GN model are identified with solutions of the s-mKdVh. In Sec. III, the s-KdVh and one useful Lax pair formulation of it are summarized, showing their relation to symmetries in quantum mechanics. How to obtain s-mKdVh solutions starting from s-KdVh solutions will also be shown. In Sec. IV, the contents of the previous section are connected to CrumDarboux spectral transformations, and their relation to supersymmetric quantum mechanics is introduced. In Sec. V, algebro-geometric/finite-gap solutions of the $\mathrm{s}-\mathrm{KdVh}$ are presented in Its-Matveev form. Through Crum-Darboux transformations, infinite families of finitegap potential with soliton defects are obtained. Two types of spectral transformations are studied from finite-gap potentials, which in Sec. VI, will be related to three types of solutions of the GN model with different spectral characteristics. Finally, in Sec. VII, the self-consistency conditions of the stationary condensates of the GN model will be studied. As an example, the condensate with solitonic potential well defects on a two-gap background will be detailed.

\section{THE GN MODEL, SERIES EXPANSION OF THE GORKOV RESOLVENT AND THE s-mKdVh}

The GN model is described by the Lagrangian

$$
\mathcal{L}_{\mathrm{GN}}=\sum_{j=1}^{N} \bar{\psi}_{j} i ð \psi_{j}+\frac{g^{2}}{2}\left(\sum_{j=1}^{N} \bar{\psi}_{j} \psi_{j}\right)^{2},
$$

where $\psi_{j}$, for $j=1,2, \ldots, N$, correspond to $N$ fermions of different flavors. A bosonization is allowed for this model, where the bosonic field corresponds to the fermionic condensate $\Delta=-g^{2}\left(\sum_{j=1}^{N} \bar{\psi}_{j} \psi_{j}\right)$.

Through the path integral approach, an effective action $S_{\text {eff }}$ for $\Delta$ can be obtained,

$$
S_{\mathrm{eff}}=-\int \frac{\Delta^{2}}{2 g^{2}} d^{2} x-i N \ln \operatorname{det}[i \not \partial-\Delta] .
$$

At the 't Hooft limit, where $N \rightarrow \infty$ and $g^{2} N \sim 1$, it is possible to use the saddle point method to ensure the convergence of the two-point propagator associated with (2.1). The convergence happens for the minimums of (2.2). The variation of such an action for the stationary case yields the following consistency equations:

$$
\Delta(x)=-i N g^{2} \operatorname{tr}_{D, \mathcal{E}}\left[\gamma^{0} R(x ; \mathcal{E})\right],
$$

where $R(x ; \mathcal{E}) \equiv\left\langle x\left|\left(H^{D}-\mathcal{E}\right)^{-1}\right| x\right\rangle$ is a $2 \times 2$ matrix known as the Gorkov diagonal resolvent of the Bogoliubov-de Gennes operator or Dirac Hamiltonian in $1+1 \mathrm{D}$,

$$
H^{D}=\left(\begin{array}{cc}
-i \frac{d}{d x} & \Delta(x) \\
\Delta(x) & i \frac{d}{d x}
\end{array}\right), \quad H^{D} \Psi=\mathcal{E} \Psi,
$$

$\gamma_{0}=\sigma_{1}$. Thus, the solutions of (2.3) correspond to the semiclassical solutions of the GN model. At this point, it is possible to connect with the Hartree-Fock treatment of the GN model, where the consistence equation for the condensate $\Delta$ is related to the population of fermion flavors in the form

$$
\Delta(x)=-g^{2}\left(\sum_{\mathrm{occ}} \bar{\psi} \psi\right)
$$

where $\sum_{\text {occ }}$ corresponds to the sum over all occupied states in the spectra of (2.4), taking into account the flavor degeneration.

By exploiting the properties of the resolvent in (2.3), a general approach to construct analytical solutions for the model was found $[10,11]$. The resolvent $R(x ; \mathcal{E})$ satisfies the following algebraic properties $R=R^{\dagger}, \operatorname{tr}_{D}\left(R \sigma_{3}\right)=0$, $\operatorname{det} R=-\frac{1}{4}$ and also satisfies the Dickey-Eilenberger equation [21]

$$
\frac{\partial}{\partial x} R \sigma_{3}=i\left[\left(\begin{array}{cc}
\mathcal{E} & -\Delta \\
\Delta & -\mathcal{E}
\end{array}\right), R \sigma_{3}\right]
$$

The power series expansion of the Gorkov resolvent on the energy variable, $R=\sum_{n}^{\infty} r_{n}(x) / \mathcal{E}^{n}$, can be truncated in order to find analytic solutions for the condensate $\Delta(x)$. In this case the resolvent takes the form

$R_{n}(x ; \mathcal{E})=\mathcal{N}(\mathcal{E}) \sum_{l=0}^{n} \mathcal{E}^{n-l}\left(\begin{array}{cc}\hat{g}_{l}(x) & \hat{f}_{l-1}(x) \\ \hat{f}_{l-1}^{*}(x) & \hat{g}_{l}(x)\end{array}\right)$,

under the condition

$$
\hat{f}_{n}=0
$$

and the latter is known as the $n$th equation of the s-mKdVh, where $\hat{g}_{l}(x)$ and $\hat{f}_{l}(x)$ are completely defined by (2.6) in the following recursive form: 


$$
\begin{aligned}
\hat{f}_{l} & =-\frac{i}{2} \hat{f}_{l-1}^{\prime}+\Delta \hat{g}_{l}, \\
\hat{g}_{l} & =i \int\left(\hat{f}_{l-1}-\Delta \hat{f}_{l-1}^{*}\right) d x+c_{l}^{D}, \\
\hat{f}_{-1} & =0, \quad \hat{f}_{0}=\Delta(x), \quad \hat{g}_{0}=c_{0}^{D}=1,
\end{aligned}
$$

where $c_{l}^{D}$ are real integration constants, $c_{2 j+1}^{D}=0$, $j, l \in \mathbb{N}_{0}$.

The truncation condition (2.8) defines $\Delta(x)$ as a solution of the s-mKdVh. The first five equations in the hierarchy correspond to

$$
\begin{aligned}
\hat{f}_{-1}(x) & =0, \\
\hat{f}_{0}(x) & =\Delta(x), \\
\hat{f}_{1}(x) & =-\frac{i}{2} \Delta^{\prime}, \\
\hat{f}_{2}(x) & =-\frac{1}{4}\left(\Delta^{\prime \prime}-2 \Delta^{3}\right)+c_{2}^{D} \Delta, \\
\hat{f}_{3}(x) & =\frac{i}{8}\left(\Delta^{\prime \prime \prime}-6 \Delta^{2} \Delta^{\prime}\right)-\frac{i c_{2}^{D}}{2} \Delta^{\prime} .
\end{aligned}
$$

This hierarchy of equations corresponds to integrable systems and can be solved by algebro-geometric methods. This is because the equations in the s-mKdVh allow Lax pair formulation, which corresponds to write (2.8) as two commutating operators $\left[H^{D}, P^{D}\right]=0$, where $P^{D}$ is a $2 \times 2$ matrix differential operator of order $n$ and takes the role of the Lax-Novikov integral of the $H^{D}$ Dirac Hamiltonian. For the s-mKdVh, the Lax integral $P^{D}$ takes the form

$$
P_{n}^{D}=\sum_{\ell=0}^{n}\left(\begin{array}{cc}
\hat{g}_{\ell}(x) & \hat{f}_{\ell-1}(x) \\
\hat{f}_{\ell-1}^{*}(x) & \hat{g}_{\ell}(x)
\end{array}\right) \sigma_{3} H^{D n-\ell},
$$

for which the Lax equation in the stationary case is

$$
\left[P_{n}^{D}, H^{D}\right]=\left(\begin{array}{cc}
0 & 2 \hat{f}_{n}(x) \\
-2 \hat{f}_{n}^{*}(x) & 0
\end{array}\right)=\mathbf{0} .
$$

This formulation relates stationary solutions $\Delta(x)$ of the $\mathrm{mKdVh}$ to scalar potentials for one-dimensional Dirac Hamiltonian operators that have the Lax-Novikov integral of motion. In addition, the interpretation of those Dirac Hamiltonians as Bogoliubov-de Gennes (2.4) operators relates the $\Delta(x)$ potentials to the stationary solutions of the GN model.

The coefficients $c_{k}^{D}$ are related to the edges of the spectrum of Hamiltonian operator $H^{D}$,

$$
\begin{aligned}
\sigma\left(H^{D}\right)= & \left(-\infty, \mathcal{E}_{0}\right] \cup\left[\mathcal{E}_{1}, \mathcal{E}_{2}\right] \cup \cdots \\
& \cup\left[\mathcal{E}_{2 j-1}, \mathcal{E}_{2 j}\right] \cup\left[\mathcal{E}_{2 n+1}, \infty\right),
\end{aligned}
$$

$\mathcal{E}_{2 j-1} \leq \mathcal{E}_{2 j}$, in the form

$$
c_{k}^{D}=\sum_{\substack{j_{0} j_{1}, \ldots, j_{n}=0 \\ j_{0}+j_{1}+\cdots+j_{n}=k}}^{k} 2^{-2 k} \prod_{i=0}^{2 n+1} \frac{\left(2 j_{i}\right) !}{\left(j_{i} !\right)^{2}\left(2 j_{i}-1\right)}\left(\mathcal{E}_{i}\right)^{j_{i}}
$$

The self-consistency equation (2.3) corresponds to a system of equations that defines the occupation of each physical state of the spectra of $H^{D}$ by the different flavors.

Another important behavior of the Lax pair operators is a Burchnall-Chaundy type relationship between matrix differential operators, which relates powers of the Lax pair operators in the following form:

$$
P_{n}^{D 2}=\mathcal{P}_{n, B C}\left(H^{D}\right)=\prod_{\ell=0}^{2 n-1}\left(H^{D}-\mathcal{E}_{\ell}\right),
$$

which defines the eigenvalues $z^{D}$ of $H^{D}$ and $y^{D}$ of $P^{D}$ over a hyperelliptic curve

$$
\left(y^{D}\right)^{2}=\prod_{\ell=0}^{2 n-1}\left(z^{D}-\mathcal{E}_{\ell}\right) .
$$

The latter relation is in the basis of the algebro-geometric solution method for the s-mKdVh.

The normalization constant $\mathcal{N}(\mathcal{E})$ of the diagonal resolvent (2.7) is defined by the condition $\operatorname{det} R=-\frac{1}{4}$ as follows:

$$
\operatorname{Det}\left(R_{n}(x ; \mathcal{E})\right)=\mathcal{N}(\mathcal{E})^{2} \mathcal{P}_{n, B C}(\mathcal{E})=-\frac{1}{4}
$$

\section{THE s-KdVh, SYMMETRIES IN QUANTUM MECHANICS, AND s-KdVh $\rightleftharpoons$ s-mKdVh MIURA TRANSFORMATION}

The supersymmetric method of construction of new condensates for the GN model is based on the Miura transformation between solutions of the $\mathrm{mKdVh}$ and the $\mathrm{KdVh}$. In order to introduce this transformation, first it is necessary to summarize the s-KdVh, and then the way to obtain solutions of the s-mKdVh from pairs of solutions of the $\mathrm{s}-\mathrm{KdVh}$ will be shown.

The s-KdVh corresponds to a set of nonlinear integrable systems, and its equations are recursively defined as follows:

$$
f_{\ell, x}=-\frac{1}{4} f_{\ell-1, x x x}+u f_{\ell-1, x}+\frac{1}{2} u_{x} f_{\ell-1},
$$

$f_{0}=1$, being the equations of the $\mathrm{s}-\mathrm{KdVh}$

$$
2 f_{\ell, x}=0
$$

Explicitly, one finds 


$$
\begin{aligned}
2 f_{0, x}= & 0 \\
2 f_{1, x}= & u_{x} \\
2 f_{2, x}= & -\frac{1}{4}\left(u_{x x x}-6 u u_{x}-4 c_{1} u_{x}\right) \\
2 f_{3, x}= & \frac{1}{8}\left(16 u_{x x x x}-5 u_{x} u_{x x}-5 u_{x}^{2}-5 u u_{x x}\right. \\
& +15 u^{2} u_{x}-2 c_{1}\left(u_{x x x}-6 u u_{x}\right) \\
& \left.+8 c_{2} u_{x}\right)
\end{aligned}
$$

where $c_{\ell}$ are real valued integration constants.

These equations allow Lax pair formulation in the form

$$
2 f_{\ell+1, x}=i\left[P_{2 \ell+1}, H\right],
$$

where $[.,$.$] is the usual commutator;$

$$
H=H(u)=-\frac{d^{2}}{d x^{2}}+u
$$

corresponds to the stationary Schrödinger operator in one dimension, $\hbar=2 m=1$; the operator

$$
\begin{aligned}
P_{2 \ell+1} & =P_{2 \ell+1}(u, \partial \sigma(H(u))) \\
& =-i \sum_{j=1}^{\ell}\left(f_{\ell-j} \frac{d}{d x}-\frac{1}{2} f_{\ell-j, x}\right) H^{\ell}
\end{aligned}
$$

is called the Lax operator; and $\partial \sigma(H(u))$ is the border of the physical spectrum of $H(u)$ that defines the coefficients $c_{j}$ in $P_{2 \ell+1}$.

The Lax equation $\left[H, P_{2 \ell+1}\right]=0$ describes an odd order integral of motion for each Schrödinger Hamiltonian associated with solutions $u$ of the s-KdVh. The order of this integral, called the Lax-Novikov integral, depends on the number of allowed bands and bound states in the spectrum of such a Hamiltonian.

The constants $c_{j}$ in (3.3) are defined as function of the energies $E_{m} \in \partial \sigma(H(u))$,

$$
c_{k}=-\sum_{\substack{j_{0}, j_{1}, \ldots, j_{2}=0 \\ j_{0}+j_{1}+\cdots+j_{2 g}=k}}^{k} 2^{-2 k} \prod_{i=0}^{2 g} \frac{\left(2 j_{i}\right) !}{\left(j_{i} !\right)^{2}\left(2 j_{i}-1\right)} E_{i}^{j_{i}},
$$

where $k=1, \ldots, \ell$ and $c_{0}=1$.

There exists a transformation between the $\mathrm{mKdVh}$ solutions and the KdVh solutions, which is called the Miura transformation. The Miura transformation is defined by

$$
u=v^{2}-v_{x} .
$$

If $v$ is any s-mKdVh solution, there is a $\hat{f}_{2 n-1}$ such that

$$
\hat{f}_{2 n-1}(v)=0 .
$$

Then due to the identity

$$
f_{n, x}(u)=i\left(2 v-\partial_{x}\right) \hat{f}_{2 n-1}(v),
$$

$u$ is a $\mathrm{s}-\mathrm{KdVh}$ solution,

$$
f_{n, x}(u)=0 .
$$

Note that the inverse affirmation is not correct.

The s-mKdVh is invariant under the change $v \rightarrow-v$, and hence the transformation (3.7) allows one to define

$$
u^{+}=v^{2}+v_{x}
$$

and

$$
u^{-}=v^{2}-v_{x},
$$

where $u^{ \pm}$are both s-KdVh solutions dependent on $v$. From another perspective: let two functions $u^{+}(x)$ and $u^{-}(x)$ satisfy the same equation in s-KdVh and depend on a function $v(x)$ as in (3.11) and (3.12), respectively. In this case, $v(x)$ must simultaneously satisfy (3.9) for $v$ and for $-v$. By adding these two equations the following identity is obtained: $4 v \hat{f}_{i}=0$, which implies that $v$ must satisfy $\mathrm{s}-\mathrm{mKdV}$ equation (3.8). This frame is in the basis of the hidden supersymmetry of the stationary sector of the GN model. It is natural to ask, why change the problem from the search of one solution of the $\mathrm{mKdVh}$ to the search of two connected solutions of the KdVh? In the next sections, it is shown how, by starting from an initial Schrödinger potential $u^{+}$and its eigenstates, it is possible to construct through the Darboux transformation a supersymmetric pair $u^{-}$and a superpotential $v(x)$. A characteristic of the Darboux transformation is to keep the symmetries, so if $u^{+}$has a Lax-Novikov integral (i.e., a s-KdVh solution), then $u^{-}$also has its respective LaxNovikov integral (i.e., another s-KdVh solution). By observing intertwining operators that generate such transformations $\left[A=\frac{d}{d x}+v(x)\right]$, families of superpotentials $v(x)$ will be found, which will be solutions of the $\mathrm{s}-\mathrm{mKdVh}$ and candidates to self-consistent stationary condensates of the Gross-Neveu model.

\section{CRUM-DARBOUX TRANSFORMATIONS AND SUPERSYMMETRY IN 1 + 1D}

The system of Eqs. (3.11) and (3.12) hides a supersymmetry in its structure. From the point of view of the Lax pair formulation, $u^{+}$defines the Schrödinger Hamiltonian $H^{+}=-\frac{d^{2}}{d x^{2}}+u^{+}$and $u^{-}$defines the Schrödinger Hamiltonian $H^{-}=-\frac{d^{2}}{d x^{2}}+u^{-}$, while $v$ defines the Dirac operator $H^{D}=-i \frac{d}{d x} \sigma_{3}+v \sigma_{1}$. To simplify, a unitary 
transformation to $H^{D}$ is done, which defines the operator $\mathcal{Q}_{1}=e^{-i \frac{\pi}{4} \sigma_{1}} H^{D} e^{i \frac{\pi}{4} \sigma_{1}}$, such that

$$
\mathcal{Q}_{1}=\left(\begin{array}{cc}
0 & A \\
A^{\dagger} & 0
\end{array}\right)=\left(\begin{array}{cc}
0 & \frac{d}{d x}+v \\
-\frac{d}{d x}+v & 0
\end{array}\right),
$$

and this operator $Q_{1}$ plays the role of the square root of

$$
\begin{aligned}
\mathcal{H}-E_{\star} & =\left(\begin{array}{cc}
H^{+} & 0 \\
0 & H^{-}
\end{array}\right) \\
& =-\frac{d^{2}}{d x^{2}}+v^{2}+\sigma_{3} v_{x} \\
& =\mathcal{Q}_{1}^{2}=\frac{1}{2}\left\{\mathcal{Q}_{1}, \mathcal{Q}_{1}\right\} .
\end{aligned}
$$

The operator $\mathcal{H}$ can be interpreted as an extended Schrödinger operator and corresponds to the Witten Hamiltonian of supersymmetric quantum mechanics in $1+1 \mathrm{D}$. The Hamiltonian $\mathcal{H}$ presents two fermionic integrals in the form $\mathcal{Q}_{1}$ and $\mathcal{Q}_{2}=i \sigma_{3} \mathcal{Q}_{1}$ for the grading operator $\Gamma=\sigma_{3}$. The Lie superalgebra of these integrals of motion takes the following form:

$$
\left[\mathcal{H}, \mathcal{Q}_{a}\right]=0,\left\{\mathcal{Q}_{a}, \mathcal{Q}_{b}\right\}=2 \delta_{a b}\left(\mathcal{H}-E_{\star}\right),
$$

where $a, b=1,2$.

Besides, it is possible to observe that $\Psi^{ \pm}(x)=$ $\exp \left( \pm \int_{x_{0}}^{x} \mathrm{~d} x^{\prime} v\left(x^{\prime}\right)\right)$ are eigenstates (not necessarily physicals) of $H_{ \pm}$with energy $E_{\star}$, or equivalently

$$
H^{ \pm} \Psi^{ \pm}(x)=E_{\star} \Psi^{ \pm}(x) .
$$

Starting from the state $\Psi^{ \pm}(x)$ of $H^{ \pm}, u^{\mp}(x)$ is defined as a Darboux transformation of $u^{ \pm}(x)$ in the form

$$
u^{ \pm}(x) \rightarrow u^{\mp}(x)=u^{ \pm}(x)-2\left(\ln \left(\Psi^{ \pm}(x)\right)\right)^{\prime \prime},
$$

and this type of transformations plays an important role in the theory of integrable systems, spectral analysis, and soliton systems. In the context of such transformations, the components of the fermionic integrals $\mathcal{Q}$, defined in (4.1), $A=\frac{d}{d x}+v(x)$ and $A^{\dagger}=-\frac{d}{d x}+v(x)$ (Hermitian conjugated of $A$ ), are known as intertwining operators between $H^{+}$and $H^{-}$. Because of the factorizations $H^{+}-E_{\star}=A A^{\dagger}$ and $H^{-}-E_{\star}=A^{\dagger} A$ the following intertwining relations are fulfilled:

$$
A H^{-}=H^{+} A, \quad A^{\dagger} H^{+}=H^{-} A^{\dagger} .
$$

These identities play a fundamental role in the solution of spectral problems of high complexity, since these allow one to obtain the spectrum of $H^{\mp}$ from the spectrum of $H^{ \pm}$, and vice versa. If $\Psi^{ \pm}(x, E)$ is a state of $H^{ \pm}$with energy $E$, then the intertwining relations (4.6) imply that $A^{\dagger} \Psi^{+}(x, E)$ is a state of $H^{-}$with energy $E$, while $A$ performs the reverse mapping, and $A \Psi^{-}(x, E)$ is a state of $H^{+}$with energy $E$. There will be some problems with the mapping of the states $\Psi^{ \pm}(x)$, since $A$ can be written as

$$
A=\Psi^{-}(x) \frac{d}{d x} \frac{1}{\Psi^{-}(x)},
$$

so it annihilates $\Psi^{-}(x)$ among the eigenstates of $H^{-}$, i.e., $A \Psi^{-}(x)=0$, while $A^{\dagger}$ annihilates the state $\Psi^{+}(x)$, of the same energy, among the eigenstate spectrum of $H^{+}$. In fact, by definition

$$
A^{\dagger}=-\Psi^{+}(x) \frac{d}{d x} \frac{1}{\Psi^{+}(x)},
$$

$\Psi^{+}(x)=\frac{1}{\Psi^{-}(x)}$, and $A^{\dagger} \Psi^{+}(x)=0$. At this point, it is interesting to note that if $\Psi^{ \pm}(x)$ is a concave (convex) state without zeros for $x \in \mathbb{R}$ then $\Psi^{\mp}(x)$ is a bounded eigenstate of $H^{\mp}$. In this case $u^{\mp}(x)$ shows a solitonic defect in the form of a potential well, which supports such a bound state.

Darboux transformation is generalized by the CrumDarboux transformation [22,23]. Such a transformation corresponds to the application of successive Darboux transformations and induces a formulation of nonlinear supersymmetry in quantum mechanics. An order $n$ CrumDarboux transformation to Schrödinger operator $H_{0}=$ $-\frac{d^{2}}{d x^{2}}+V_{0}(x)$ results in a new operator

$$
\begin{aligned}
& H_{n}=-\frac{d^{2}}{d x^{2}}+V_{n}(x), \\
& V_{n}=V_{0}-2 \frac{d^{2}}{d x^{2}} \log \mathbb{W}_{n},
\end{aligned}
$$

where $\mathbb{W}_{n}$ is the Wronskian of $n$ formal eigenstates $\psi_{j}$ of $H_{0}, H_{0} \psi_{j}=E_{j} \psi_{j}, E_{i} \neq E_{j}$,

$$
\begin{aligned}
& \mathbb{W}_{n}=\mathbb{W}\left(\psi_{1}, \ldots, \psi_{n}\right)=\operatorname{det} \mathcal{A}, \\
& \mathcal{A}_{i j}=\frac{d^{i-1}}{d x^{i-1}} \psi_{j}, \quad i, j=1, \ldots, n
\end{aligned}
$$

The eigenstates $\Psi_{0}(x ; E) \neq \psi_{j}$ of $H_{0}, H_{0} \Psi_{0}(x ; E)=E \Psi_{0}$ $(x ; E)$ are mapped to eigenstates $\Psi_{n}(x ; E)$ of $H_{n}, H_{n} \Psi_{n}$ $(x ; E)=E \Psi_{n}(x ; E)$ through the fraction of Wronskians,

$$
\Psi_{n}(x ; E)=\frac{\mathbb{W}\left(\psi_{1}, \ldots, \psi_{n}, \Psi_{0}(E)\right)}{\mathbb{W}_{n}},
$$

where $\mathbb{W}_{0}=1$ has been chosen. It is possible to introduce the first-order differential intertwining operators 


$$
\begin{aligned}
A_{n} & =\frac{d}{d x}+\mathcal{W}_{n}, \\
\mathcal{W}_{n} & =-\frac{d}{d x} \log \mathbb{W}_{n}+\frac{d}{d x} \log \mathbb{W}_{n-1} .
\end{aligned}
$$

These operators and their conjugate factorize $H_{n-1}$ and $H_{n}$ in the form $A_{n}^{\dagger} A_{n}=H_{n-1}-E_{n}, A_{n} A_{n}^{\dagger}=H_{n}-E_{n}$, and intertwine them as follows: $A_{n} H_{n-1}=H_{n} A_{n}, A_{n}^{\dagger} H_{n}=$ $H_{n-1} A_{n}^{\dagger}$.

The intertwining operator $A_{n}$ can equivalently be represented in the form

$$
\begin{aligned}
A_{j} & =\left(A_{j-1} \cdots A_{1} \psi_{j}\right) \frac{d}{d x} \frac{1}{\left(A_{j-1} \cdots A_{1} \psi_{j}\right)} \\
& =\frac{d}{d x}-\left(\frac{d}{d x} \ln \left(A_{j-1} \cdots A_{1} \psi_{j}\right)\right),
\end{aligned}
$$

where $A_{1}=\psi_{1} \frac{d}{d x} \frac{1}{\psi_{1}}$ and $A_{j-1} \cdots A_{1} \psi_{j}$ is an eigenstate of eigenvalue $E_{j}$ for $H_{j-1}$, any other formal eigenstate $\Psi_{j-1}(E)$ of $H_{j-1}, H_{j-1} \Psi_{k-1}(E)=E \Psi_{n-1}(E)$ is mapped for $A_{j}$ in the eigenstate $\Psi_{j}(E)=A_{j} \Psi_{j-1}(E)$ of $H_{j}$ with the same eigenstate as $H_{j} \Psi_{j}(E)=E \Psi_{j}(E)$.

In this way, it is possible to intertwine $H_{n}$ and $H_{0}$ using the order $n$ intertwining operator $\mathbb{A}_{n} \equiv A_{n} \cdots A_{1}$, $\mathbb{A}_{n} H_{0}=H_{n} \mathbb{A}_{n}, \mathbb{A}_{n}^{\dagger} H_{n}=H_{0} \mathbb{A}_{n}^{\dagger}$. If $\mathbb{W}_{n} \neq 0$ and $V_{0}(x)$ is nonsingular, for $x \in \mathbb{R}$, then the extended system $\mathcal{H}=$ $\operatorname{diag}\left(H_{0}, H_{n}\right)$ is characterized by a nonlinear supersymmetry dependent on the scattering data of the eigenstates used in Crum-Darboux transformation. In the superalgebra there exist two nilpotent $Z_{2}$-odd antidiagonal supercharges $Q_{+}=\mathbb{A}_{n}^{\dagger} \sigma_{+}=\frac{1}{2}\left(Q_{2}+i Q_{1}\right)$ and $Q_{-}=\mathbb{A}_{n} \sigma_{-}=Q_{+}^{\dagger}$, $\left[Q_{ \pm}, \mathcal{H}\right]=0, Q_{ \pm}^{2}=0$, where $\sigma_{ \pm}=\frac{1}{2}\left(\sigma_{1} \pm i \sigma_{2}\right)$. These generate a nonlinear Lie superalgebra in the form $\left\{Q_{a}, Q_{b}\right\}=2 \delta_{a b} \prod_{\ell=1}^{n}\left(\mathcal{H}-E_{\ell}\right)$.

This supersymmetric representation shows a spontaneous breaking of symmetry, which depends on the spectral data of the chosen $\psi_{i}$ states. The $H(u)$ Schrödinger operator has an order two formal degeneration for each energy level, so the election of the states $\psi_{i}$ is arbitrary, and in general, it is a linear combination between the pair of linearly independent states of the same energy. There are states that through the Darboux transformations produce (i) a nonlinear phase shift in the initial potential, (ii) one defect in the initial potential with the form of a solitonic potential well, and/or (iii) singularities. In addition to the above, special elections of pairs of states that produce singularities can altogether generate one or two soliton defects, supporting bounded states in the forbidden gaps of the spectrum of the initial potential.

It is possible to differentiate between three supersymmetric frames: exact, broken, and partially broken supersymmetries. Let $H_{n}$ and $\psi_{i}$ be defined as in (4.9), the supersymmetry associated with $\mathcal{H}$ is exact if $H_{n}\left(H_{0}\right)$ has a normalizable ground state of energy $E_{i}$ lower than the energy of the ground state of $H_{0}\left(H_{n}\right)$. In this case, $\mathcal{H}$ supports a ground state in the form $\Psi_{0}=\left(0, \mathbb{A}_{n} \psi_{i}^{\star}\right)^{T}$ [ $\left.\Psi_{0}=\left(\psi_{i}, 0\right)^{T}\right]$, where $\psi_{i}^{\star}$ is a state of $H_{0}$ of energy $E_{i}$ linearly independent of $\psi_{i}$, and thus the ground state $\Psi_{0}$ is annihilated for all generators of supersymmetry $Q_{j} \Psi_{0}=0$, $j=1,2$.

The supersymmetry is broken if $H_{0}$ and $H_{n}$ both have normalizable ground states of the same energy $E_{i}$, such that $\mathcal{H}$ has two normalizable ground states $\Phi_{0,0}=\left(\sqrt{\prod_{\ell=1}^{n}\left(E_{0}-E_{\ell}\right)} \psi_{0}, 0\right)^{T}$ and $\Phi_{0, n}=\left(0, \mathbb{A}_{n} \psi_{0}\right)^{T}$, where $\psi_{0}$ is the bound state of $H_{0}$. In this case the generators of supersymmetry do not annihilate the ground states; rather they transform them one into the other $Q_{-} \Psi_{0,0}=\sqrt{\prod_{\ell=1}^{n}\left(E_{0}-E_{\ell}\right)} \Psi_{0, n}$ and $Q_{+} \Psi_{0, n}=$ $\sqrt{\prod_{\ell=1}^{n}\left(E_{0}-E_{\ell}\right)} \Psi_{0,0}, Q_{-} \Psi_{0, n}=Q_{+} \Psi_{0,0}=0$.

A third case arises if the lower energy state in CrumDarboux transformation $\psi_{i}$ corresponds to the lower energy edge of bands, in this case $\psi_{0}=\psi_{i}$, and the supersymmetry generators annihilate both states $Q_{ \pm} \Phi_{0, a}=0, a=0, n$. About the latter case, it is necessary to say that the finitegap structure of $V_{0}$ expands the number of supersymmetry generators from $N=2$ to $N=4$. There is a central extension of the superalgebra due to the Lax-Novikov integral present in finite-gap systems. This is called a partially broken supersymmetry because the initial two fermionic integrals annihilate the two bound states while the additional two do not [24].

The central extension is also possible in the first two cases but it is essential in the definition of the third one.

An important behavior occurs when the Crum-Darboux transformation takes place, for it makes it possible to preserve the symmetries of the initial system. For example, let $H$ be a Hamiltonian with integral of motion $P$, $[H, P]=0$; a Crum-Darboux transformation to $H$ allows one to define a Hamiltonian $H^{\prime}$ and an intertwining operator $A$ between $H$ and $H^{\prime}, A H=H^{\prime} A$ and $A^{\dagger} H^{\prime}=H A^{\dagger}$, and then through Darboux dressing to $P$ it results that $P^{\prime}=A P A^{\dagger}$ is an integral of $H^{\prime}$. In simple steps it is proved that $\left[H^{\prime}, P^{\prime}\right]=0$, which ensures that by means of the Crum-Darboux transformation a solution of the $\mathrm{s}-\mathrm{KdVh}([H, P]=0)$ yields another solution of the $\mathrm{s}-\mathrm{KdVh}$ $\left(\left[H^{\prime}, P^{\prime}\right]=0\right)$. The order of the equation in the hierarchy solved by the transformed system depends on the order of $P^{\prime}$, and it can be equal, lower, or higher than the initial solution order. This is possible due to an order reduction mechanism that relies on the spectral data of $H$ and the states used in the Crum-Darboux transformation. In this direction, sometimes it is possible to reduce the operator $P^{\prime}$ to a lower order operator $\tilde{P}^{\prime}$ due to an identity in the form $P^{\prime}=\prod_{i}\left(H-\lambda_{i}\right) \tilde{P}^{\prime}$ which ensures that if $P^{\prime}$ is an integral, then $\tilde{P}^{\prime}$ also is. On the other hand, if $u$ is a solution of one equation of the $\mathrm{s}-\mathrm{KdVh}$, then automatically it is a solution of infinite equations of a higher order of the hierarchy. 
In other words, if $u$ is a solution of any equation of the hierarchy that defines a Lax pair $H$ and $P,[H, P]=0$, then it is always possible to construct a new Lax pair $H$ and $\tilde{P}=P \prod_{j=1}^{n}\left(H-\lambda_{j}\right),[H, \tilde{P}]=0$, where $\lambda_{j} \in \mathbb{R}$ and $n$ is a positive integer, that corresponds to the Lax pair formulation of a higher order equation in $\mathrm{s}-\mathrm{KdVh}$.

\section{ALGEBRO-GEOMETRIC SOLUTIONS OF THE S-KdVh AND THEIR CRUM-DARBOUX TRANSFORMATIONS}

In this section the Its-Matveev formula of the algebrogeometric finite-gap solutions of the s-KdVh is presented. Moreover, two types of spectral transformations are studied, and infinite families of finite-gap potential with solitonic defects are obtained.

The Lax pair of KdVh $H_{g, 0}=H\left(u_{g, 0}\right)$ and $P_{2 g+1}=$ $P_{2 g+1}\left(u_{g, 0}, \partial \sigma\left(H_{g, 0}\right)\right)$ satisfy the Burchnall-Chaundy relationship [25]

$$
P_{2 g+1}^{2}=\prod_{i=0}^{2 g}\left(H-E_{i}\right)
$$

which relates the eigenvalues $y$ of $P_{2 g+1}$ to the eigenvalues $z$ of $H_{g, 0}$ through the hyperelliptic curve

$$
y^{2}=\prod_{i=0}^{2 g}\left(z-E_{i}\right)
$$

The algebro-geometrical method allows one to find the finite-gap solutions in the form of second derivatives of logarithms of Riemann theta functions. The Its-Matveev formula $[26,27]$ for potentials with $g$ gaps is given by

$$
u_{g, 0}(x)=-2 \frac{d^{2}}{d x^{2}} \ln (\theta(x \mathbf{v}+\boldsymbol{\phi}, \tau))+\Lambda_{0},
$$

with $\mathbf{v}, \boldsymbol{\phi} \in \mathbb{C}^{g}$.

The eigenstates for the Hamiltonian associated with (5.3) are given in the form

$$
\psi(r, x)=\frac{\theta(x \mathbf{v}+\boldsymbol{\phi}+\boldsymbol{\alpha}(r), \tau)}{\theta(x \mathbf{v}+\boldsymbol{\phi}, \tau)} \exp (-i x \xi(r)),
$$

where $\theta$ is the Riemann theta function of genus $g$ which presents a periodicity in the form $\theta(\mathbf{z}+\mathbf{a}, \tau)=\theta(\mathbf{z}, \tau)$, for $\mathbf{a} \in \mathbb{Z}^{g}$,

$$
\theta(\mathbf{z}, \tau)=\sum_{\mathbf{n} \in \mathbb{Z}^{g}} \exp (2 \pi i\langle\mathbf{n}, \mathbf{z}\rangle+\pi i\langle\mathbf{n}, \mathbf{n} \tau\rangle),
$$

with $\mathbf{z} \in \mathbb{C}^{g}$ and $\tau$ the modular matrix. The genus $g$ of the Riemann theta function corresponds to the number of band gaps in the spectrum of the associated Schrödinger operator $[27,28]$.
The parameters in (5.3) and (5.4) are completely defined by the curve (5.2). The modular matrix is a $g \times g$ symmetric matrix with a positive defined imaginary part, whose elements, as well as the components of $\mathbf{v}$ and the constant $\Lambda_{0}$, are uniquely determined by the energies of the edges of the spectrum of $H$, while $\alpha(r)$ and $\xi(r)$ also depend on a point $r=(z, y)$ on the hyperelliptic curve (5.2) such that $H \psi(r, x)=z \psi(r, x)$ and $P_{2 g+1} \psi(r, x)=y \psi(r, x)$. On the other hand, $\phi$ depends on the full spectral data of $H$ [27].

Crum-Darboux transformations to solutions in the Its-Matveev form correspond to finite-gap systems with bound states in their forbidden bands. An equation in $\mathrm{s}-\mathrm{KdVh} 2 f_{g+l+1, x}\left(u_{g, l}(x)\right)=0$ with parameters $c_{\ell}$, $\ell=0, \ldots, 2 g+2 l$, defined by the energies $\partial \sigma\left(H_{g, l}\right)=$ $\left\{E_{0}, \ldots, E_{2 g}\right\} \cup\left(\cup_{i=1, \ldots, l}\left\{z\left(r_{i, 1}\right), z\left(r_{i, 1}\right)\right\}\right)$, has solutions with irreducible $P_{2 g+2 l+1}$ when $u_{g, l}$ takes the form

$$
\begin{aligned}
u_{g, l}(x)= & u_{g, 0}(x)-2 \frac{d^{2}}{d x^{2}} \ln \left(\mathbb { W } \left(\psi_{a_{1,1}, a_{1,2}}\left(r_{1,1}, r_{1,2}, x\right),\right.\right. \\
& \left.\left.\ldots, \psi_{a_{l, 1}, a_{l, 2}}\left(r_{l, 1}, r_{l, 2}, x\right)\right)\right),
\end{aligned}
$$

with

$\psi_{a_{i, 1}, a_{i, 2}}\left(r_{i, 1}, r_{i, 2}, x\right)=a_{i, 1} \psi\left(r_{i, 1}, x\right)+a_{i, 2} \psi\left(r_{i, 2}, x\right)$

real functions, where $a_{i, 1}$ and $a_{i, 2}$ are $\mathbb{C}$ constants and $r_{i, 1}$ and $r_{i, 2}$ are elements in different charts of the Riemann surface related to hyperelliptic curve (5.2) with $z\left(r_{i, 1}\right)=z\left(r_{i, 2}\right)$ and $y\left(r_{i, 1}\right)=-y\left(r_{i, 2}\right), z_{i} \neq z_{j}$, for $i \neq j, i, j=1, \ldots, l$.

The s-KdVh solution $u_{g, l}(x)$ defines a Lax pair in the form

$$
\begin{aligned}
H_{g, l} & =H\left(u_{g, l}(x)\right), \\
P_{2 g+2 l+1} & =P_{2 g+2 l+1}\left(u_{g, l}(x), \partial \sigma\left(H_{g, l}\right)\right),
\end{aligned}
$$

where the Darboux dressing of the integral $P_{2 g+1}$ yields the identity $P_{2 g+2 l+1}=\mathbb{A}_{l} P_{2 g+1} \mathbb{A}_{l}^{\dagger}, \mathbb{A}_{l}=A_{l} A_{l-1} \cdots A_{1}$ with $A_{j}$ defined as in (4.12) but changing $\psi_{i} \rightarrow \psi_{a_{i, 1}, a_{i, 2}}\left(r_{i, 1}, r_{i, 2}, x\right)$.

To perform a quantum mechanical interpretation, the operator $H$ must fulfill the role of Hamiltonian and $P_{2 g+2 l+1}$ the role of integral of motion. To ensure real valued observables, it must be required that both $H$ and $P$ be Hermitian operators without singularities in the real axis. ${ }^{1}$ It is necessary to demand that $u_{g, l}(x)$ have no singularities for $x \in \mathbb{R}$ and that its spectrum be real, which implies $E_{i}$, $z_{j}, \Lambda_{0} \in \mathbb{R}$, for $i=0, \ldots, 2 g$ and $j=1, \ldots, l$. Under these conditions the operation $\dagger$ corresponds to the Hermitian conjugation.

\footnotetext{
${ }^{1}$ It is not necessary to require this interval as an Hermiticity condition (an example of this is the infinite potential well) but, given the nature of solitonic potentials, this is the most natural choice for the present type of systems.
} 
To construct $u_{g, l}(x)$ nonsingular, it is possible to choose $u_{g, 0}(x)$ and $u_{g, l}(x)-u_{g, 0}(x)$ both nonsingular or both singular but in the latter case the singularities of the first term must cancel the singularities of the second. To obtain $u_{g, 0}(x)$ singular, it is enough with a correct election of $\phi$. Overall, this is possible when $z_{i} \in \sigma(H)^{c}$, where $c$ corresponds to the complement; i.e., $\psi_{a_{i}, a_{2}}\left(r_{i, 1}, r_{i, 2}, x\right)$ must be nonphysical states of $H$. It is necessary to use the zeros theorem for the correct choice of sign of the ratio $a_{i, 1} / a_{i, 2}$ for each energy $z\left(r_{i, 1}\right)$. Note that there are an infinite number of solutions.

Soliton potential wells added by Crum-Darboux transformations to the solution in Its-Matveev form deform the shape of the initial finite-gap potential. It is possible to obtain different shape types of solitonic potential wells depending on which forbidden band supports the associated bounded states. The asymptotic behaviors of the Crum-Darboux transformation correspond to phase shifts in the periodic structures of the initial potential.

\section{A. Types of Miura-Darboux transformations}

Two types of Darboux transformations to $u_{g, l}(x)$ are interesting. Their differences produce three types of solutions for the GN model.

Auto Darboux transformations: the real eigenstates $\mathbb{A}_{l} \psi(r, x)$ for $H_{g, l}, z(r) \leq E_{i}, E_{i} \in \partial \sigma\left(H_{g, l}\right)$, correspond to nonsingular states of modulated exponential growing (decreasing), which cannot generate bound states through Darboux transformation. As a consequence of the ItsMatveev formula, the Darboux transformation using $\mathbb{A}_{l} \psi(r, x)$ connects two shape invariant isospectral potentials, $u_{g, l}(x)$ and $\tilde{u}_{g, l}^{r}(x)$. The potential $\tilde{u}_{g, l}^{r}(x)$ differs from $u_{g, l}(x)$ in phase shifts of the positions of the solitons (due to changes in the coefficients $a_{l, i}$ ) and of the crystal structures that generate each band. The phase shift in crystal structures is given as follows:

$$
\delta \phi=\tilde{\boldsymbol{\phi}}^{r}-\boldsymbol{\phi}=\boldsymbol{\alpha}(r),
$$

where $\boldsymbol{\phi}$ is defined in (5.3) and $\boldsymbol{\alpha}(r)$ comes from the definition of $\psi(r, x)$ in (5.4). $\boldsymbol{\phi}$ and $\tilde{\boldsymbol{\phi}}^{r}$ correspond to vectors whose components are the phases of the crystal structures in $u_{g, l}(x)$ and $\tilde{u}_{g, l}^{r}(x)$, respectively.

The operators

$$
\begin{aligned}
X_{l}(r) & =\mathbb{A}_{l} \psi(r, x) \frac{d}{d x} \frac{1}{\mathbb{A}_{l} \psi(r, x)}, \\
X_{l}(r)^{\dagger} & =-\frac{1}{\mathbb{A}_{l} \psi(r, x)} \frac{d}{d x} \mathbb{A}_{l} \psi(r, x)
\end{aligned}
$$

intertwine $H_{g, l}=X_{l}(r)^{\dagger} X_{l}(r)+z(r)$ and $\tilde{H}_{g, l}^{r}=X_{l}(r)$ $X_{l}(r)^{\dagger}+z(r)=-\frac{d^{2}}{d x^{2}}+\tilde{u}^{r}(x)$ in the form $X_{l}(r) H_{g, l}=$ $\tilde{H}_{g, l}^{r} X_{l}(r)$ and $X_{l}(r)^{\dagger} \tilde{H}_{g, l}^{r}=H_{g, l} X_{l}(r)^{\dagger}$, respectively. By being the initial and the transformed potentials isospectral by construction, these must be solutions of the same equation of the s-KdVh with exactly the same coefficients $c_{\ell}$. Thus, $\tilde{H}^{r}$ must have a Lax-Novikov integral $\tilde{P}_{2 g+2 l+1}^{r}=$ $P_{2 g+2 l+1}\left(\tilde{u}_{g, l}^{r}(x), \partial \sigma\left(H_{g, l}\right)\right)$, such that $\left[\tilde{P}_{2 g+2 l+1}^{r}, \tilde{H}_{g, l}^{r}\right]=$ $-2 i \frac{d}{d x} f_{g+l+1}\left(\tilde{u}^{r}(x), \partial \sigma\left(H_{g, l}\right)\right)$. It is possible to connect the Lax operators through the intertwining operator (5.10) in the form

$$
X(r) P_{2 g+1}=\tilde{P}_{2 g+1}^{r} X(r) .
$$

To prove this identity, it is enough to note that $X(r)$ $P_{2 g+1}\left(X(r) P_{2 g+1}\right)^{\dagger}=\tilde{P}_{2 g+1}^{r} X(r)\left(\tilde{P}_{2 g+1}^{r} X(r)\right)^{\dagger}$ and that any deformation of (5.12) in the form $X(r) P_{2 g+1}=$ $\tilde{P}_{2 g+1}^{r} X(r)+D(r)$, with $D(r)$ an intertwining operator between $H_{g, l}$ and $\tilde{H}_{g, l}^{r}$, is inconsistent for any order of $D(r)$.

The simplest example of auto Darboux transformation corresponds to choosing $\psi(r, x)$ as the generator of the Darboux transformations to $u_{g, 0}(x)$. The result of such a transformation is

$$
\tilde{u}_{g, 0}^{r}(x)=-2 \frac{d^{2}}{d x^{2}} \ln \left(\theta\left(x \mathbf{v}+\tilde{\boldsymbol{\phi}}^{r}, \tau\right)\right)+\Lambda_{0},
$$

a shape invariant transformation of $u_{g, 0}(x)$ with $\phi$ displaced to $\tilde{\boldsymbol{\phi}}^{r}=\boldsymbol{\phi}+\boldsymbol{\alpha}(r)$.

Solitonic Darboux transformations: these are Darboux transformations constructed using real states of $H_{g, l-1}$ of the form $\Psi[l]=\mathbb{A}_{l-1} \psi_{a_{l, 1}, a_{l, 2}}\left(r_{l, 1}, r_{l, 2}, x\right)$, where $\mathbb{A}_{l-1}$, $\psi_{a_{l, 1}, a_{l, 2}}\left(r_{l, 1}, r_{l, 2}, x\right)$, are defined in (5.7) and the paragraphs below it.

The intertwining operators

$$
\begin{aligned}
& A_{l}=\Psi[l] \frac{d}{d x} \frac{1}{\Psi[l]}, \\
& A_{l}^{\dagger}=-\frac{1}{\Psi[l]} \frac{d}{d x} \Psi[l]
\end{aligned}
$$

intertwine $\quad H_{g, l-1}=A_{l}^{\dagger} A_{l}+z\left(r_{l, 1}\right) \quad$ with $\quad H_{g, l}=A_{l} A_{l}^{\dagger}+$ $z\left(r_{l, 1}\right)$, in the form $A_{l} H_{g, l-1}=H_{g, l} A_{l}$ and $A_{l}^{\dagger} H_{g, l}=$ $H_{g, l-1} A_{l}^{\dagger}$. The Darboux dressing of the Lax-Novikov integral $P_{2 g+2(l-1)+1}$ of $H_{g, l-1}$ allows one to find a LaxNovikov integral $\hat{P}_{2 g+2 l+1}=A_{l} P_{2 g+2(l-1)+1} A_{l}^{\dagger}$ for $H_{g, l}$, such that $\left[P_{2 g+2 l+1}, H_{g, l}\right]=0$. Since $P_{2 g+2 l+1}$ is two orders greater than $P_{2 g+2(l-1)+1}$, it is possible to note that $u_{g, l}$ is a solution of an equation in $\mathrm{s}-\mathrm{KdVh}$ of one order higher than the initial solution $u_{g, l-1}$.

In $[16,24]$ the solitonic potentials and their respective Lax-Novikov integrals for the free background and the Lamé periodic background have been studied in detail, and concrete norms for the construction of solitonic potentials have been established from the zeros theorem. The simplest example of solitonic Darboux transformation 
is to use $\psi_{a 1,1, a 1,2}\left(r_{1,1}, r_{1,2}, x\right)$ as the generator of a Darboux transformation to $u_{g, 0}(x)$. The result of such a transformation is

$$
\begin{aligned}
u_{g, 1}(x)= & -2 \frac{d^{2}}{d x^{2}} \ln \left(a_{1,1} \theta\left(x \mathbf{v}+\boldsymbol{\phi}+\boldsymbol{\alpha}\left(r_{1,1}\right), \tau\right)\right. \\
& \times \exp \left(-i x \xi\left(r_{1,1}\right)\right)+a_{1,2} \theta\left(x \mathbf{v}+\boldsymbol{\phi}+\boldsymbol{\alpha}\left(r_{1,2}\right), \tau\right) \\
& \left.\times \exp \left(-i x \xi\left(r_{1,2}\right)\right)\right)+\Lambda_{0},
\end{aligned}
$$

where the constants $\xi\left(r_{j, 1}\right)$ and $\xi\left(r_{j, 2}\right)$ present an imaginary part different from zero for $z\left(r_{j, 1}\right)$ in the forbidden bands of the spectrum of $H_{g, 0}$; this is due to the exponential growth or decrease of nonphysical states. Far from the center of the soliton defect, the solitonic Darboux transformation looks like an auto Darboux transformation because only nonlinear phase shifts remain in the transformed potential. This is due to the asymptotic dominance of one of the exponential terms in (5.15). Both asymptotic potentials correspond to potentials in the Its-Matveev form with phase shifts $\left|\Delta \boldsymbol{\phi}_{i}\right|=\left|\boldsymbol{\alpha}_{i}\left(r_{1,2}\right)-\boldsymbol{\alpha}_{i}\left(r_{1,1}\right)\right|$, where $i=1, \ldots, g$ corresponds to the index of each vector component.

\section{STATIONARY CONDENSATES FOR THE GN MODEL}

It is possible to differentiate between three types of stationary solutions for the GN model in dependence of their shapes and the spectrum of their respective Dirac operators: (i) kink finite-gap condensates: in this case, the condensate oscillates around zero and presents a central allowed band [see Figs. 1(a) and 2(a)]; (ii) kink-antikink finite-gap condensates: in this case the shape of the condensate oscillates around a constant different from zero and presents a central gap [see Figs. 1(b) and 2(b)]; and (iii) kink on kink-antikink finite-gap condensates: these condensates are associated with solitonic Darboux transformations, present a domain wall that divides two different kink-antikink finite-gap phases. Such a domain wall is supported by a single bound state of zero energy [see Figs. 1(c) and 2(c)]. Solitonic defects with Dirac energies closer than zero allow one to construct pairs of domain walls [see Fig. 3].



(a)

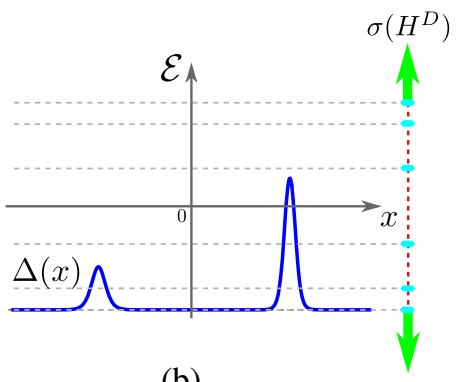

(b)

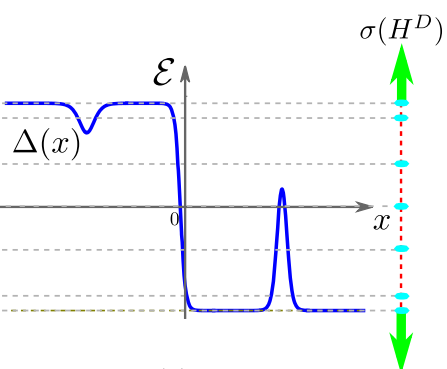

(c)

FIG. 1. Examples of stationary condensates of the GN model: solitonic defects on a homogeneous background. For each graphic are shown the shape of the condensate $\Delta(x)$, in a blue continuous line, and its spectrum $\sigma\left(H^{D}\right)$, in green thick lines the continuous spectrum, in dashed red line the band gaps, and in cyan dots the bound states and the band edges. Note that $-\Delta(x)$ is a condensate solution of the GN model too, with an identical spectrum. (a) Trivial zero solution, (b) homogeneous background with two kink-antikink defects, and (c) kink on a homogeneous background with two kink-antikink defects.

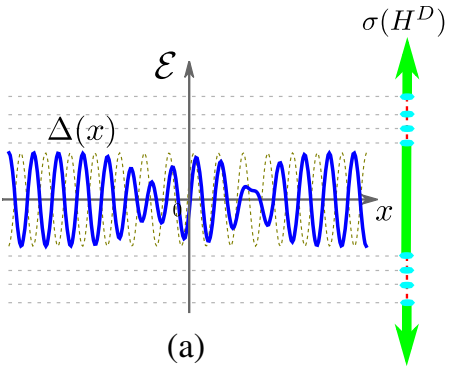

(a)

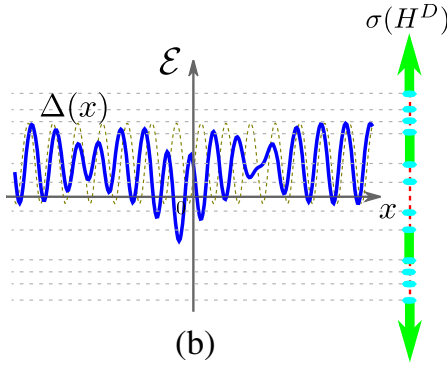

(b)

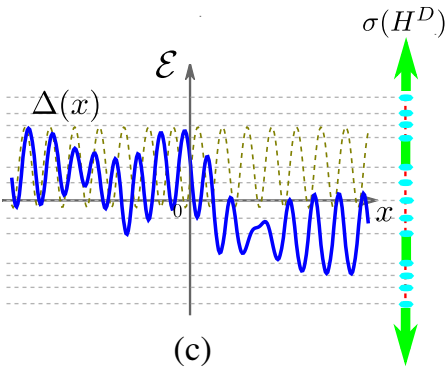

(c)

FIG. 2. Examples of stationary condensates of the GN model: solitonic defects on two- and three-gap scalar Dirac potentials. The same symbology as in Fig. 1 is used but with a brown dashed thin line added to show the shape of the finite-gap background: (a) a two-gap condensate with two modulation shape solitonic defects that support each two bound states in the external gaps, one in each forbidden band; (b) a three-gap background with three solitonic defects, two of them as modulations and one kink-antikink defect that supports two bound states in the central band gap; and (c) a kink version of the previous condensate, where its spectrum additionally contains a zero energy state. 


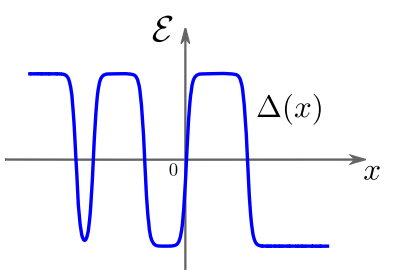

(a)

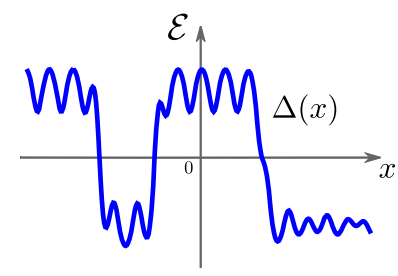

(b)
FIG. 3. Kink defects look like a domain wall that pushes away two different phases of the condensate, while soliton defects with energies very close to zero allow one to have two transitions between these two phases, which is due to the spontaneous appearance of a kink and an antikink. The width of the kinkantikink increases while the energy of the defect approaches zero, while in the zero limit one or both domain walls can disappear in the spatial infinities.

\section{A. Defects on kink-antikink finite-gap condensates}

Through auto Darboux transformations

$$
\Delta=-\frac{d}{d x} \ln \left(\mathbb{A}_{l} \psi\left(r_{\star}\right)\right)
$$

are constructed. The pair of Miura transformations that defines $\Delta$ in (6.1) corresponds to $u_{g, l}-z_{\star}=\Delta^{2}-\frac{d}{d x} \Delta$ and $\tilde{u}_{g, l}^{r_{\star}}-z_{\star}=\Delta^{2}+\frac{d}{d x} \Delta$.

The described transformation allows one to define the extended Schrödinger Hamiltonian $\tilde{\mathcal{H}}=\operatorname{diag}\left(H_{g, l}, \tilde{H}_{g, l}^{r_{*}}\right)$, the fermionic integrals

$$
\tilde{\mathcal{Q}}_{1}=\left(\begin{array}{cc}
0 & X_{1, l}\left(r_{\star}\right)^{\dagger} \\
X_{1, l}\left(r_{\star}\right) & 0
\end{array}\right)=\left(\begin{array}{cc}
0 & -\frac{d}{d x}+\Delta \\
\frac{d}{d x}+\Delta & 0
\end{array}\right),
$$

and $\tilde{\mathcal{Q}}_{2}=i \sigma_{3} \tilde{\mathcal{Q}}_{1}$, and also a Lax-Novikov integral in the form

$$
\tilde{\mathcal{P}}_{1}=\left(\begin{array}{cc}
P_{2 g+2 l+1} & 0 \\
0 & \tilde{P}_{2 g+2 l+1}^{r_{\star}}
\end{array}\right)
$$

that satisfies the following superalgebra:

$$
\begin{gathered}
{\left[\tilde{\mathcal{H}}, \tilde{\mathcal{Q}}_{a}\right]=0, \quad\left\{\tilde{\mathcal{Q}}_{a}, \tilde{\mathcal{Q}}_{b}\right\}=2 \delta_{a b}\left(\tilde{\mathcal{H}}-z_{\star}\right),} \\
{\left[\tilde{\mathcal{P}}_{1}, \tilde{\mathcal{H}}\right]=\mathbf{0}, \quad\left[\tilde{\mathcal{P}}_{1}, \mathcal{Q}_{a}\right]=\mathbf{0} .}
\end{gathered}
$$

The identities (6.5) correspond to the Lax pair formulation of the equations of both $\mathrm{s}-\mathrm{KdVh}$ and $\mathrm{s}-\mathrm{mKdVh}$, respectively. Remember that the equations in the $\mathrm{mKdVh}$ are invariant under the change $\Delta \rightarrow-\Delta$; hence $\Delta=$ $\frac{d}{d x} \ln \left(\mathbb{A}_{l} \psi\left(r_{\star}\right)\right)$ also corresponds to a stationary GN condensate.

Additionally, it is possible to define $\tilde{\mathcal{P}}_{2}=\sigma_{3} \tilde{\mathcal{P}}_{1}$, which is another Lax-Novikov integral for $\tilde{\mathcal{H}},\left[\tilde{\mathcal{P}}_{2}, \tilde{\mathcal{H}}\right]=\mathbf{0}$. The integral $\tilde{\mathcal{P}}_{2}$ together with $\tilde{\mathcal{Q}}_{a}, a=1,2$ defines a pair of new fermionic integrals $\left[\tilde{\mathcal{P}}_{2}, \tilde{\mathcal{Q}}_{a}\right]$ that complete the $N=4$ fermionic integrals.

As a consequence of (6.5), the kink-antikink condensates $\Delta$ described in (6.1) are solutions of $\hat{f}_{2 g+2 l+1}(\Delta)=0$ [see (2.10)], where coefficients $c_{k}^{D}$ are given by the energies $\partial \sigma\left(\tilde{\mathcal{Q}}_{1}\right)$,

$$
\begin{aligned}
\sigma\left(\tilde{\mathcal{Q}}_{1}\right)= & \left(-\infty,-\sqrt{E_{0}-z_{\star}}\right] \cup\left[-\sqrt{E_{1}-z_{\star}},-\sqrt{E_{2}-z_{\star}}\right] \cup \cdots \cup\left[-\sqrt{E_{2 g-1}-z_{\star}},-\sqrt{E_{2 g}-z_{\star}}\right] \\
& \cup\left[\sqrt{E_{2 g}-z_{\star}}, \sqrt{E_{2 g-1}-z_{\star}}\right] \cup \cdots \cup\left[\sqrt{E_{2}-z_{\star}}, \sqrt{E_{1}-z_{\star}}\right] \cup\left[\sqrt{E_{0}-z_{\star}}, \infty\right) \\
& \cup\left(-\sqrt{z_{j}-z_{\star}}, \sqrt{z_{j}-z_{\star}}\right\},
\end{aligned}
$$

where $\left\{E_{0}, \ldots, E_{2 n}\right\}=\partial \sigma\left(H_{g, 0}\right)$ and $z_{j}=z\left(r_{j, 1}\right)=z\left(r_{j, 2}\right)$ the energies of the bound states supported by the solitons in the potential of $H_{g, l}$. Note that for a common base of eigenstates the eigenvalues $\tilde{\mathcal{E}}$ of $\tilde{\mathcal{Q}}_{1}$ and the eigenvalues $\tilde{E}$ of $\tilde{\mathcal{H}}$ are related in the form $\tilde{\mathcal{E}}^{2}=\tilde{E}-z_{\star}$. This type of solutions, as seen in Fig. 2(b), corresponds to self-consistent condensates $\Delta$ with a central energy band gap.

\section{B. Defects on kink finite-gap condensates}

These are the limits of kink-antikink finite-gap condensates in which $\mathbb{A}_{l} \psi\left(r_{\star}\right)$ is the ground state of $H_{g, l}$, and it must also correspond to an edge of band, with energy $E_{2 g}$.
In this case $P_{2 g+2 l+1}$ and $X_{1, l}\left(r_{\star}\right)$ share $\mathbb{A}_{l} \psi\left(r_{\star}\right)$ as a kernel, and then there exist $S$ such that $P_{2 g+2 l+1}=S X_{1, l}\left(r_{\star}\right)$ and $\tilde{P}_{2 g+2 l+1}^{r_{\star}}=X_{1, l}\left(r_{\star}\right)^{\dagger} S^{\dagger}$. This produces an order reduction of the Lax-Novikov central integral of the Dirac Hamiltonian operator $\tilde{\mathcal{Q}}_{1}$, where $\tilde{\mathcal{P}}_{1}=\tilde{\mathcal{Q}}_{1} \tilde{\mathcal{S}}_{1}$,

$$
\tilde{\mathcal{S}}_{1}=\left(\begin{array}{cc}
0 & S^{\dagger} \\
S & 0
\end{array}\right), \quad\left[\tilde{\mathcal{S}}_{1}, \tilde{\mathcal{Q}}_{1}\right]=0 .
$$

The identity (5.12) defines $\tilde{\mathcal{S}}_{1}$ and $\tilde{\mathcal{Q}}_{1}$ as the Lax pair of $\hat{f}_{2 g+2 l}(\Delta)=0$ in the s-mKdVh, with coefficients $c_{k}^{D}$ given by the boundaries of the spectrum of $\tilde{\mathcal{Q}}_{1}, \partial \sigma\left(\tilde{\mathcal{Q}}_{1}\right)$. 


$$
\begin{aligned}
\sigma\left(\tilde{\mathcal{Q}}_{1}\right)= & \left(-\infty,-\sqrt{E_{0}-E_{2 g}}\right] \cup\left[-\sqrt{E_{1}-E_{2 g}},-\sqrt{E_{2}-E_{2 g}}\right] \cup \cdots \cup\left[-\sqrt{E_{2 g-1}-E_{2 g}}, \sqrt{E_{2 g-1}-E_{2 g}}\right] \\
& \cup \cdots \cup\left[\sqrt{E_{2}-E_{2 g}}, \sqrt{E_{1}-E_{2 g}}\right] \cup\left[\sqrt{E_{0}-E_{2 g}}, \infty\right) \cup \cup_{j=1}^{l}\left\{-\sqrt{z_{j}-E_{2 g}}, \sqrt{z_{j}-E_{2 g}}\right\} .
\end{aligned}
$$

In this case $\tilde{\mathcal{Q}}_{a}, \tilde{\mathcal{S}}_{1}$ and $\tilde{\mathcal{S}}_{2}=i \sigma_{3} \tilde{\mathcal{S}}_{1}$ correspond to the four fermionic integrals of $\tilde{\mathcal{H}}$.

\section{Soliton defects plus kink on kink-antikink finite-gap background}

Through solitonic Darboux transformations

$$
\Delta=-\frac{d}{d x} \ln \left(\mathbb{A}_{l-1} \psi_{a_{l, 1}, a_{l, 2}}\left(r_{l, 1}, r_{l, 2}, x\right)\right)
$$

are constructed. In this case, the pair of satisfied Miura transformations are $u_{g, l-1}-z_{l}=\Delta^{2}-\frac{d}{d x} \Delta$ and $u_{g, l}-z_{l}=$ $\Delta^{2}+\frac{d}{d x} \Delta$.

The extended Schrödinger Hamiltonian takes the form $\mathcal{H}=\operatorname{diag}\left(H_{g, l-1}, H_{g, l}\right)$ and its Lax-Novikov integral can be written as

$$
\mathcal{P}_{1}=\left(\begin{array}{cc}
\left(H_{g, l-1}-z_{l}\right) P_{2 g+2(l-1)+1} & 0 \\
0 & P_{2 g+2 l+1}
\end{array}\right),
$$

$\left[\mathcal{P}_{1}, \mathcal{H}\right]=0$. In the definition of $\mathcal{P}_{1}$ has been introduced the term $\left(H_{g, l-1}-z_{l}\right)$ to have operators of the same order in its diagonal elements, which will be necessary in the next analysis.

In this case, it is easy to show that $\mathcal{P}_{1}$ plays the role of integral of motion for the Dirac Hamiltonian operators

$$
\begin{gathered}
\mathcal{Q}_{1}=\left(\begin{array}{cc}
0 & A_{l}^{\dagger} \\
A_{l} & 0
\end{array}\right), \quad \mathcal{Q}_{2}=i \sigma_{3} \mathcal{Q}_{1}, \\
{\left[\mathcal{P}_{1}, \mathcal{Q}_{a}\right]=0 .}
\end{gathered}
$$

On the other hand, in the Dirac Hamiltonian frame the kink nature of the superpotential in $\mathcal{Q}_{a}$ generates a spontaneous order reduction of the s-mKdVh Lax-Novikov operator $\mathcal{P}_{1}$ because in this case it is possible to do the factorization $\mathcal{P}_{1}=\mathcal{Q}_{1} \mathcal{S}_{1}$,

$$
\begin{aligned}
& \mathcal{S}_{1}=\left(\begin{array}{cc}
0 & P_{2 g+2(l-1)+1} A_{l}^{\dagger} \\
A_{l} P_{2 g+2(l-1)+1} & 0
\end{array}\right), \\
& \mathcal{S}_{2}=i \sigma_{3} \mathcal{S}_{1},
\end{aligned}
$$

and from this point of view, it is allowed the order reduction $\mathcal{P}_{1} \rightarrow \mathcal{S}_{1}$. So taking $\mathcal{Q}_{1}$ as the Dirac Hamiltonian, the irreducible integral of motion, in the form of the s-mKdV Lax-Novikov operator (2.10), is not $\mathcal{P}_{1}$ but rather $\mathcal{S}_{1}$,

$$
\left[\mathcal{Q}_{1}, \mathcal{S}_{1}\right]=0 \text {. }
$$

$\Delta$ are the solutions of $\hat{f}_{2 g+2 l}(\Delta)=0$ with coefficients $c_{k}^{D}$ given by the boundaries of the spectrum of $\mathcal{Q}_{1}, \partial \sigma\left(\mathcal{Q}_{1}\right)$.

$$
\begin{aligned}
\sigma\left(\mathcal{Q}_{1}\right)= & \left(-\infty,-\sqrt{E_{0}-z_{l}}\right] \cup\left[-\sqrt{E_{1}-z_{l}},-\sqrt{E_{2}-z_{l}}\right] \cup \cdots \cup\left[-\sqrt{E_{2 n-1}-z_{l}},-\sqrt{E_{2 n}-z_{l}}\right] \\
& \cup 0 \cup\left[\sqrt{E_{2 n}-z_{l}}, \sqrt{E_{2 n-1}-z_{l}}\right] \cup \cdots \cup\left[\sqrt{E_{2}-z_{l}}, \sqrt{E_{1}-z_{l}}\right] \cup\left[\sqrt{E_{0}-z_{l}}, \infty\right) \\
& \left.\cup j-\sqrt{z_{j}-z_{l}}, \sqrt{z_{j}-z_{l}}\right\} .
\end{aligned}
$$

The existence of a zero energy state is directly related to the kink or antikink nature of $\Delta$.

For a common base of states the energies $\mathcal{E}$ of $\breve{\mathcal{Q}}=\mathcal{Q}_{1}, \tilde{\mathcal{Q}}_{\star}$ and the energies $E$ of $\breve{\mathcal{H}}=\mathcal{H}, \tilde{\mathcal{H}}$ keep the relation $\mathcal{E}^{2}=$ $E-\breve{z}$, where $\breve{z}$ correspond to $z_{l}$ or $z_{\star}$, respectively. The stationary condensates $\Delta$ have the characteristic property that their spectra are always symmetrical with respect to $\mathcal{E}=0$.

\section{SOLITONIC DEFECTS AND SELF-CONSISTENCY}

The role of the consistence equations (2.7) is to define the spectrum of fermion matter that composes each condensate. There exist infinite possibilities to fill the fermion states in the spectrum of a condensate $\Delta$. Among them, it is possible to differentiate between ground states and exciton solutions. In ground state configurations, a particle (hole) occupies (empties) a state only if those of smaller (greater) energy are also occupied (emptied), while exciton configurations allow discontinuities in the occupation of the states.

By using the identities (2.7) and (2.15), the consistency equation (2.3) for a condensate $\Delta$ with $n+1$ gaps and $2 l$ defects, $n, l \in \mathbb{N}_{0}$, takes the form

$$
\begin{aligned}
i \frac{\Delta}{N g^{2}} & =\operatorname{tr}_{\mathcal{E}}\left[\frac{\mathcal{E}^{n+2 l} \Delta+\Sigma}{\sqrt{-\Pi} \prod_{k=1}^{l}\left(\mathcal{E}^{2}-\mathcal{E}_{b, k}^{2}\right)}\right], \\
\Sigma & =\sum_{j=1}^{\lfloor n / 2\rfloor+l} \mathcal{E}^{n+2 l-2 j} \hat{f}_{2 j}, \\
\sqrt{-\Pi} & =\sqrt{-\prod_{j=0}^{2 n+1}\left(\mathcal{E}-\mathcal{E}_{e, j}\right),}
\end{aligned}
$$




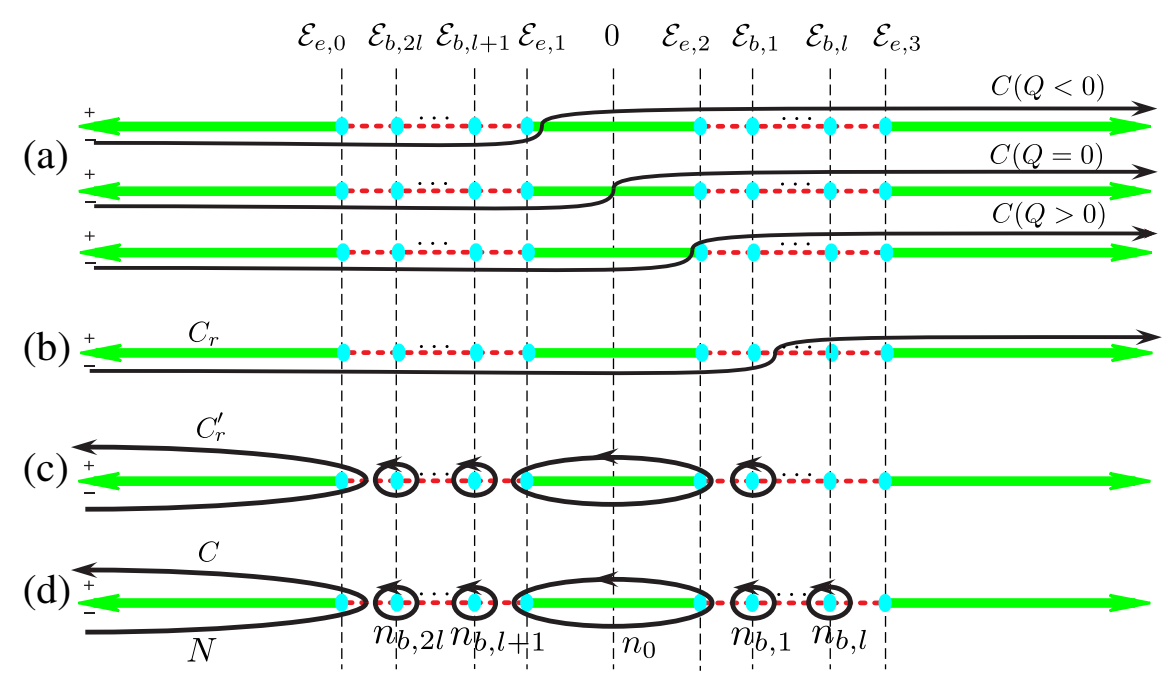

FIG. 4. Occupation of fermion matter. The occupation of the fermionic states by each flavor is characterized by the spectrum of the condensate $\Delta$. Such a spectrum defines the poles and the branch cuts in the argument of the trace in Eq. (7.1). Here the meaning of the integration paths for ground state configurations is shown: (a) Examples of paths for ground states; the first path leaves holes in Dirac sea (antiparticles) so its charge $Q$ will be "negative." The second case corresponds to a full Dirac sea, where there are no particles nor antiparticles, and thus, the total charge is zero. In the third case, there are only particles, so it has a "positive" charge. Since the paths cross the axis only once, these examples correspond to ground state type paths. (b) The path $C_{r}$ for the $r$ th fermion flavor. The path $C_{r}$ in (b) is identical to the path $C_{r}^{\prime}$ in (c). $C_{r}^{\prime}$ results from adding a closed integration path in the upper plane to $C_{r}$, and such a closed integration path covers the axis $\operatorname{Im}[z]=0$ with direction $\operatorname{Re}[z]=\infty \rightarrow \operatorname{Re}[z]=-\infty$ and whose integral, by means of the residue theorem, is equal to zero. (d) The superposition of the integration path of all flavors construct the path $C$. To simplify, the scattering zones, of particles and holes, are chosen empty. In other words, the lower allowed band is occupied by all the flavors, i.e., the path $C$ cover $N$ times the respective branch cut, and the upper allowed band is empty. On the other hand, some flavors occupy the bound states, and then the poles are turned by $C$ a number $n<N$ of times. Another assumption for simplicity is to choose all the states in the bands occupied by the same number of fermions.

where $g^{2}$ is the coupling constant of the GN model, ${ }^{2}$ the constants $\mathcal{E}_{e, j}$, for $j=0,1, \ldots, 2 n+1$, correspond to the energies of the edges of the bands of $H^{D}$, and $\mathcal{E}_{b, k}=$ $-\mathcal{E}_{b, l+k}, 0<\mathcal{E}_{b, 1}<\mathcal{E}_{b, 2}<\cdots<\mathcal{E}_{b, l}$, to the energies of the bound states of $H^{D}$. Here $\operatorname{tr}_{\mathcal{E}}$ is defined as

$$
\operatorname{tr}_{\mathcal{E}} \equiv \frac{1}{N} \sum_{r=1}^{N} \int_{C_{r}} \frac{d \mathcal{E}}{2 \pi} \equiv \frac{1}{N} \int_{C} \frac{d \mathcal{E}}{2 \pi},
$$

where the integration paths $C_{r}$ in (7.2) depend on the spectrum occupied by the particles and antiparticles (holes) of flavor $r$ (see Fig. 4). Thus, Eq. (7.1) corresponds to a system of $\lfloor n / 2\rfloor+l+1$ equations that defines the UV cutoff and the occupation of each state in the spectrum of $H^{D}$ by the different flavors.

By observing that Eq. (7.1) must be fulfilled for any values of $\hat{f}_{2 j}=\hat{f}_{2 j}(x), j=1, \ldots,\lfloor n / 2\rfloor+l$, it is possible to reduce those equations in the following set of consistence equations: one equation for UV cutoff

$$
\frac{i}{N g^{2}}=\operatorname{tr}_{\mathcal{E}}\left[\frac{\mathcal{E}^{n}}{\sqrt{-\Pi}}\right]
$$

\footnotetext{
${ }^{2}$ To avoid confusion, note that in this section $g^{2}$ is not related to the genus of the Riemann theta function, for here $n$ takes this role.
}

$2\lfloor n / 2\rfloor$ equations for the occupation of the bands

$$
0=\operatorname{tr}_{\mathcal{E}}\left[\frac{\mathcal{E}^{n-j}}{\sqrt{-\Pi}}\right]
$$

where $j=1, \ldots, 2\lfloor n / 2\rfloor$, and $l$ equations for the occupation of the bound states

$$
0=\operatorname{tr}_{\mathcal{E}}\left[\frac{\mathcal{E}^{n-2\lfloor n / 2\rfloor}}{\sqrt{-\Pi}\left(\mathcal{E}^{2}-\mathcal{E}_{b, j}^{2}\right)}\right],
$$

where $j=1, \ldots, l$. The unknown variables of these equations are the occupation fractions $\nu(|\mathcal{E}|)=\frac{n(-|\mathcal{E}|)-n(|\mathcal{E}|)}{N}$, $-1<\nu(|\mathcal{E}|)<1$, where $n(\mathcal{E})$ is the number of flavors that occupy the eigenstate of allowed energy $\mathcal{E}$.

It is interesting to note that the result of (7.5) for each pair of defects is independent of the other pairs. The occupation fraction $\nu_{k}=\nu\left(\left|\mathcal{E}_{b, k}\right|\right)$ for the pair of bounded states of energies $\left|\mathcal{E}_{b, k}\right|$ and $-\left|\mathcal{E}_{b, k}\right|$ depends on the spectral information of the finite-gap background and only on the modulo of the energy of such a pair of defects, without taking into account the existence of the other pairs. This is a characteristic already observed in the solitonic case in the free massive background.

The consistence equations are the same for a kink condensate as for an identical condensate without the kink. 
In kink condensates there are $2 l+1$ bound states, where one of such states has zero energy. In Eq. (7.1) this change corresponds to multiplying the numerator and denominator by $\mathcal{E}$, the consistence equations remaining unchanged. As a consequence, the consistence equations (2.3) do not fill the occupation of the state of zero energy, and by definition $\nu(0)=0$.

In the following, the self-consistency of the condensates that present solitonic defects on one- and two-gap backgrounds will be studied. For simplicity, a ground state occupation will be presented, in which the states of the lower allowed band are fully occupied, the states in the upper allowed band are completely empty, and all the states in the allowed bands have the same occupation fraction.

The one-gap solution corresponds to massive Dirac particle $\Delta= \pm M, M>0$. In this case, Dirac operator $\mathcal{Q}$ [see (6.2)] connects two copies of $H_{0,0}=-\frac{d^{2}}{d x^{2}}$. The eigenstates of $H_{0,0}$ correspond to $\psi(k)=e^{i k x}, H_{0,0} \psi(k)=$ $E \psi(k), E=k^{2}, k \in \mathbb{R}$ for physical states and $k \in \mathbb{I}$ for nonphysical states. Through the Miura-Darboux transformation, the massive condensate rises from the state $\psi(i m)=e^{-m x}, m= \pm M$, being $\Delta=-\frac{d}{d x} \ln \psi(i m)$. Note that for $m=0$ the 0 -gap condensate is obtained through the physical edge of band state $\psi(0)=1$ of $H_{0,0}$.

The one-gap condensates with defects are obtained using nonphysical states of $H_{0,0}$ in the form

$$
\Delta=-\frac{d}{d x} \ln \left(\frac{\mathbb{W}\left(\breve{\psi}_{1}, \ldots, \breve{\psi}_{j}, e^{-m x}\right)}{\mathbb{W}\left(\breve{\psi}_{1}, \ldots, \breve{\psi}_{j}\right)}\right)
$$

where $\breve{\psi}_{2 \ell}=\sinh \left(\kappa_{2 \ell}\left(x+\tau_{2 \ell}\right)\right)$ and $\breve{\psi}_{2 \ell+1}=\cosh \left(\kappa_{2 \ell+1}\right.$ $\left.\left(x+\tau_{2 \ell+1}\right)\right)$, with $j$ and $\ell$ positive integers and $\left|\kappa_{\ell}\right|<\left|\kappa_{\ell-1}\right|<|m|$. In this case, the spectrum of $\mathcal{Q}$ corresponds to

$$
\begin{aligned}
\sigma(\mathcal{Q})= & (-\infty,-M] \cup_{i=1, \ldots, j}-\sqrt{M^{2}-\kappa_{i}^{2}} \\
& \cup_{i=1, \ldots, j} \sqrt{M^{2}-\kappa_{i}^{2}} \cup[M, \infty),
\end{aligned}
$$

and Eq. (7.5) defines the occupation fraction $\nu_{k}$ of the bound states of the central band in the form

$$
\left|\mathcal{E}_{b, j}\right|=M \sin \left(\frac{\pi \nu_{j}}{2}\right), \quad j=1, \ldots, l .
$$

Note that for this type of condensates the occupation fractions are related by $\nu_{1}<\nu_{2}<\cdots<\nu_{l}$. This result allows solutions of the ground state type, in which any particle of any flavor can exist only if there exists another of the same flavor with the allowed energy immediately lower.

Within the solutions on the finite-gap background, the inhomogeneous simplest case (modulo spatial displacements) is the kink crystal background

$$
\begin{aligned}
\Delta & =-(\ln \operatorname{dn}(M x, k))^{\prime} \\
& =M k^{2} \frac{\operatorname{sn}(M x, k) \operatorname{cn}(M x, k)}{\operatorname{dn}(M x, k)},
\end{aligned}
$$

where $\operatorname{sn}(x, k), \operatorname{cn}(x, k)$, and $\operatorname{dn}(x, k)$ are the Jacobi elliptic functions with modular parameter $k, 0<k<1$. The condensate (7.8) is a solution of the s-mKdVh,

$$
0=\Delta^{\prime \prime}(x)-2 \Delta(x)^{3}-2\left(k^{2}-2\right) M^{2} \Delta(x),
$$

and this equation is also known as the nonlinear Schrödinger equation and corresponds to $\hat{f}_{2}(\Delta)=0$.

From now on, the following relationship between Jacobi's theta function and Riemann's theta function will be used:

$$
\begin{gathered}
\Theta(x \mid k)=\theta\left(x+\frac{1}{2}, \tau\right), \quad \tau=i \frac{\mathbf{K}^{\prime}}{\mathbf{K}}, \\
\operatorname{dn}(u, k)=\frac{\Theta(u+\mathbf{K})}{\Theta(u)} \frac{\Theta(0)}{\Theta(\mathbf{K})},
\end{gathered}
$$

where $\mathbf{K}($.$) is the elliptic integral of the first kind, \mathbf{K}=$ $\mathbf{K}(k)$ is the complete elliptic integral of the first kind, and $\mathbf{K}^{\prime}=\mathbf{K}\left(k^{\prime}\right), k^{\prime}=\sqrt{1-k^{2}}$.

From the supersymmetric point of view, the kink crystal condensate connects two Lamé one-gap Schrödinger potentials displaced in half a period: $u_{1,0}=$ $M^{2} k^{2}\left(2 \operatorname{sn}(M x, k)^{2}-1\right) \quad$ and $\quad \tilde{u}_{1,0}^{(0,0)}=M^{2} k^{2}(2 \operatorname{sn}(M x+$ $\left.\mathbf{K}(k), k)^{2}-1\right)$. The Darboux transformation that generates this superpotential is constructed using the ground state $\psi=\operatorname{dn}(M x, k)$ of the one-gap Schrödinger system $H_{1,0}$. Such a Darboux transformation allows one to construct a kink two-gap condensate, with two band gaps separating a central band [29].

This condensate has a period of $2 \mathbf{K}(k)$, defined in terms of the complete elliptic integral of first kind $\mathbf{K}(k)$.

The spectrum of kink crystal (7.8) is given by $\sigma\left(H^{D}\right)=$ $(-\infty,-M] \cup\left[-M k^{\prime}, M k^{\prime}\right] \cup[M, \infty)$, where the energy band gaps correspond to $-M<\mathcal{E}<-M k^{\prime}$ and $M k^{\prime}<$ $\mathcal{E}<M$.

The eigenstates of $H_{1,0}, H_{1,0} \psi_{ \pm}^{\alpha}=E(\alpha) \psi_{ \pm}^{\alpha}$ are

$$
\psi_{ \pm}^{\alpha}(x)=\frac{\mathrm{H}(M x \pm \alpha)}{\Theta(M x)} \exp [\mp M x \mathrm{Z}(\alpha)],
$$

where $E(\alpha)=M^{2} \operatorname{dn}^{2}(\alpha, k)$ and $\mathrm{Z}$ and $\mathrm{H}$ are the Jacobi zeta and eta functions respectively,

$$
\begin{gathered}
\mathrm{Z}(u \mid k)=\frac{d}{d u} \ln \Theta(u), \\
\mathrm{H}(u)=-i q^{1 / 4} \exp \left(i \frac{\pi u}{2 \mathbf{K}}\right) \Theta\left(u+i \mathbf{K}^{\prime}\right),
\end{gathered}
$$




$$
q=\exp (i \pi \tau)
$$

For more details about these functions and the spectral properties such as quasimomentum, see [16]. The functions $\Psi_{ \pm}^{\alpha}$ are parametrized in terms of the parameter $\alpha$, which lies in a rectangular domain with vertices $\alpha \in\{0, \mathbf{K}, \mathbf{K}+$ $\left.i \mathbf{K}^{\prime}, i \mathbf{K}^{\prime}\right\}$.

By using chains of Darboux transformations, it is possible to construct an infinite set of symmetric condensates with one central allowed band in the form

$$
\begin{gathered}
\Delta(x)=-\frac{d}{d x} \ln \left(\frac{W(\phi(1), \ldots, \phi(2 \ell), \operatorname{dn}(M x, k))}{W(\phi(1), \ldots, \phi(2 \ell))}\right), \\
\phi(2 j+1) \equiv C_{2 j+1} \psi_{+}^{\alpha_{2 j+1}}(x)+\frac{1}{C_{2 j+1}} \psi^{\alpha^{2 j+1}}(x), \\
\phi(2 j) \equiv C_{2 j} \psi_{+}^{\alpha_{2 j}}(x)-\frac{1}{C_{2 j}} \psi^{\alpha_{2 j}}(x), \\
0<\alpha_{1}<\alpha_{2}<\cdots<\alpha_{2 \ell}<\mathbf{K} .
\end{gathered}
$$

The spectrum of these condensates is defined by

$$
\begin{aligned}
\sigma\left(H^{D}\right)= & (-\infty,-M] \cup_{i=1}^{2 \ell}\left\{-M \operatorname{dn}\left(\alpha_{i}, k\right)\right\} \\
& \cup\left[-M k^{\prime}, M k^{\prime}\right] \\
& \cup_{i=1}^{2 \ell}\left\{M \operatorname{dn}\left(\alpha_{i}, k\right)\right\} \cup[M, \infty) .
\end{aligned}
$$

It is possible to obtain potentials with $2(2 \ell-1)$ bound states, and one way to obtain these is to choose a parameter $C_{j}$ and to take either the limit $C_{j} \rightarrow 0$ or $\infty$. With this method, one of the solitons disappears in some of the two spatial infinities, $x \rightarrow \pm \infty$. As a consequence of the loss of two bound states, the characteristic equation in s-mKdVh of the initial condensates will fall by two orders. In summary, through this method stationary condensates $\Delta$ are constructed, whose spectra are symmetrical with respect to $\mathcal{E}=0$; they also have a finite number of bands and any number of bound states in them.

Because of the different occupation number of each sector of the spectrum, the occupation fraction $\nu_{0}$ is introduced for the occupation of the states in the central band and $\nu_{j}, j=1, \ldots, l$, for the bound states.

Because of the symmetries of the spectrum, the occupation constant $\nu_{0}$ is undefined as in the kink case. Thus, the consistency equation (7.5) for the condensate (7.15) takes the form

$$
\nu_{l}=\frac{2}{\pi} \tan ^{-1}\left(\frac{\sqrt{\mathcal{E}_{b, l}^{2}-k^{\prime 2} M^{2}}}{\sqrt{M^{2}-\mathcal{E}_{b, l}^{2}}}\right),
$$

and the result shows how the effective occupation fraction of the defects relies on their energy and width of the central band (or the same, the modular parameter $k$ ). The inverse interpretation defines the energy of the defect as a function of the effective occupation fraction and the width of the central band

$$
\left|\mathcal{E}_{b, l}\right|=M \cos \left(\frac{\pi \nu_{1}}{2}\right) \sqrt{k^{\prime 2}+\tan ^{2}\left(\frac{\pi \nu_{1}}{2}\right)} .
$$

It is interesting to notice that in limit $k^{\prime} \rightarrow 0$ known results of the solitonic case in the free massive background are obtained (7.7). In this limit $k \rightarrow 1, \mathbf{K} \rightarrow \infty$, and the kink crystal (7.8) is reduced to the Callan-Coleman-Gross-Zee kink $\Delta=M \tanh (M x)$ [12].

For more complex condensates, the consistence equations depend on hyperelliptic integrals of higher order.

\section{DISCUSSION AND OUTLOOK}

By using an exotic supersymmetry between finite-gap systems with defects, the set of analytical stationary solutions for the GN model has been constructed, observing the existence of inhomogeneous and nonperiodic condensates with band structures and a finite number of bound states.

The Darboux transformation has allowed the recursive construction of infinite families of exactly solvable Scrödinger systems from a finite-gap potential given by the Its-Matveev formula.

The process of constructing solitary defects on finite-gap Schrödinger potentials has generated scalar Dirac potentials (or Bogoliubov-de Gennes self-consistent condensates) that present solitary defects on finite-gap backgrounds. Each one of these Dirac systems exhibits an irreducible integral of motion corresponding to a Lax operator of the s-mKdVh.

The Darboux dressing of the Lax operator of finite-gap systems has allowed the finding of the self-consistency equations for all the stationary condensates, having as a main characteristic the independence of the consistence equations for each pair of bound states of opposite energy, each one depending only on finite-gap background data and the modulus of its characteristic energy.

On the dependence of their shape and spectrum, the set of self-consistent stationary condensates presented here can be separated into three groups. These condensates necessarily have the form of (i) kink finite-gap condensates with solitonic defects, which oscillate around zero and present a central allowed band, (ii) kink-antikink finite-gap condensates with solitonic defects, which oscillate around a constant different from zero and present a central forbidden band, and (iii) kink domain wall condensates on kinkantikink finite-gap background with solitonic defects, which looks like a kink (antikink) domain wall that pushes 
away two kink-antikink finite-gap phases and supports one bound state of zero energy in the central forbidden band; the two kink-antikink finite-gap phases oscillate around opposite constants.

In what follows, some interesting related study problems will be discussed. Important aspects to be studied about the condensates presented here are their mass spectrum and their stability. In the direction of the first point, the study of the Green function and the density of states of the respective Schrödinger and Dirac equations for finite-gap systems with defects are within the research interests of the author. About the stability of these condensates and their decay channels [17]: the possible decays of allowed bands into bound states or allowed bands into other allowed bands are mysteries yet to be revealed.

The thermodynamic role that these condensates could occupy in a GN model is another interesting point of study, the most direct approach being the GinzburgLandau expansion of the thermodynamic grand potential $11,15,18]]$.

On the other hand, as well as s-mKdVh is related to the GN model, the Zakharov-Shabat Ablowitz-Kaup-NewellSegur hierarchy (ZS-AKNS) is related to the chiral GN model or the Nambu-Jona-Lasinio model in $1+1 \mathrm{D}$. There exists a generalization of the Darboux transformation for Dirac operators in $1+1 \mathrm{D}$. Particularly, such a transformation allows the construction of pseudoscalar
Dirac potentials in the form of soliton defects on finitegap backgrounds with the spectrum not necessarily symmetric. Although these potentials also present a Lax-Novikov integral, they are not solutions of the s$\mathrm{mKdVh}$, rather of the ZS-AKNS hierarchy of equations [30,31]. In general, these potentials correspond to complex pseudoscalar potentials with asymmetric spectra, any number of bound states, and a finite number of energy band gaps. Because of the existence of the LaxNovikov integral and an adequate Darboux transformation, it is possible to find a representation for a nonlinear $N=4$ supersymmetry for extended Dirac Hamiltonians.

The supersymmetric method has proved to be useful for solving problems of nonlinear interaction between bosons $[32,33]$ and problems of nonlinear interaction between fermions, herein studied. In this direction, it is interesting to search for uses of the supersymmetric methods for the study of coupled systems between bosons and fermions.

\section{ACKNOWLEDGMENTS}

The author is thankful to Dr. Mikhail Plyushchay and Dr. Mokhtar Hassaine for critical comments and to Roberto Muñoz, Claudia Baeza, and Guillermo Guerra for their kind help and hospitality. The research was partially supported from FONDECYT through Grant No. 3170474.
[1] D. J. Gross and A. Neveu, Dynamical symmetry breaking in asymptotically free field theories, Phys. Rev. D 10, 3235 (1974).

[2] Y. Nambu and G. Jona-Lasinio, Dynamical model of elementary particles based on an analogy with supercoductivity. 1., Phys. Rev. 122, 345 (1961).

[3] K. Rajagopal and F. Wilczek, The condensed matter physics of QCD, in At the Frontier of Particle Physics: Handbook of $Q C D$, edited by M. Shifman (World Scientific, Singapore, 2001).

[4] P. Fulde and R. A. Ferrell, Superconductivity in a strong spin-exchange field, Phys. Rev. 135, A550 (1964); A. I. Larkin and Y. N. Ovchinnikov, Nonuniform state of superconductors, Zh. Eksp. Teor. Fiz. 47, 1136 (1964) [Sov. Phys. JETP 20, 762 (1965)].

[5] R. Peierls, The Quantum Theory of Solids (Oxford University Press, Oxford, UK, 1955); P. G. de Gennes, Superconductivity of Metals and Alloys (Addison-Wesley, Redwood City, CA, 1989).

[6] R. Casalbuoni and G. Nardulli, Inhomogeneous superconductivity in condensed matter and QCD, Rev. Mod. Phys. 76, 263 (2004).

[7] A. J. Heeger, S. Kivelson, J. R. Schrieffer, and W.-P. Su, Solitons in conducting polymers, Rev. Mod. Phys. 60, 781 (1988).
[8] D. K. Campbell and A. R. Bishop, Solitons in polyacetylene and relativistic-field-theory models, Phys. Rev. B 24, 4859 (1981).

[9] A. Saxena and A. R. Bishop, Multipolaron solutions of the Gross-Neveu field theory: Toda potential and doped polymers, Phys. Rev. A 44, R2251 (1991).

[10] G. Basar and G. V. Dunne, Self-Consistent Crystalline Condensate in Chiral Gross-Neveu and Bogoliubov-de Gennes Systems, Phys. Rev. Lett. 100, 200404 (2008); Twisted kink crystal in the chiral Gross-Neveu model, Phys. Rev. D 78, 065022 (2008); Gross-Neveu models, nonlinear Dirac equations, surfaces and strings, J. High Energy Phys. 01 (2011) 127.

[11] G. Basar, G. V. Dunne, and M. Thies, Inhomogeneous condensates in the thermodynamics of the chiral $\mathrm{NJL}_{2}$ model, Phys. Rev. D 79, 105012 (2009).

[12] R. F. Dashen, B. Hasslacher, and A. Neveu, Semiclassical bound states in an asymptotically free theory, Phys. Rev. D 12, 2443 (1975).

[13] J. Feinberg and S. Hillel, Stable fermion bag solitons in the massive Gross-Neveu model: Inverse scattering analysis, Phys. Rev. D 72, 105009 (2005); Fermion bag solitons in the massive Gross-Neveu and massive Nambu-Jona-Lasinio models in $1+1$ dimensions: Inverse scattering analysis, J. Phys. A 39, 21 (2006). 
[14] G. Dunne and J. Feinberg, Self-isospectral periodic potentials and supersymmetric quantum mechanics, Phys. Rev. D 57, 1271 (1998).

[15] F. Correa, G. V. Dunne, and M. S. Plyushchay, The Bogoliubov-de Gennes system, the AKNS hierarchy, and nonlinear quantum mechanical supersymmetry, Ann. Phys. (N.Y.) 324, 2522 (2009).

[16] A. Arancibia, J. M. Guilarte, and M. S. Plyushchay, Effect of scalings and translations on the supersymmetric quantum mechanical structure of soliton systems, Phys. Rev. D 87, 045009 (2013).

[17] J. Feinberg, On kinks and bound states in the Gross-Neveu model, Phys. Rev. D 51, 4503 (1995); The periodic table of static fermion bags in the Gross-Neveu model, Int. J. Mod. Phys. A 17, 898 (2002); All about the static fermion bags in the Gross-Neveu model, Ann. Phys. (N.Y.) 309, 166 (2004).

[18] M. Thies, Analytical solution of the Gross-Neveu model at finite density, Phys. Rev. D 69, 067703 (2004), and references therein; From relativistic quantum fields to condensed matter and back again: Updating the GrossNeveu phase diagram, J. Phys. A 39, 12707 (2006).

[19] G. V. Dunne and M. Thies, Time-Dependent Hartree-Fock Solution of Gross-Neveu Models: Twisted-Kink Constituents of Baryons and Breathers, Phys. Rev. Lett. 111, 121602 (2013).

[20] G. V. Dunne and M. Thies, Full time-dependent HartreeFock solution of large $N$ Gross-Neveu models, Phys. Rev. D 89, 025008 (2014).

[21] I. Kosztin, S. Kos, M. Stone, and A. J. Leggett, Free energy of an inhomogeneous superconductor: A wave-function approach, Phys. Rev. B 58, 9365 (1998); S. Kos and M. Stone, Gradient expansion for the free energy of a clean superconductor, Phys. Rev. B 59, 9545 (1999).

[22] M. M. Crum, Associated Sturm-Liouville systems, Quart. J. Math. Oxford Ser. (2) 6, 121 (1955).
[23] V. B. Matveev and M. A. Salle, Darboux Transformations and Solitons (Springer, Berlin, 1991).

[24] A. Arancibia, F. Correa, V. Jakubsky, J. M. Guilarte, and M.S. Plyushchay, Soliton defects in one-gap periodic system and exotic supersymmetry, Phys. Rev. D 90, 125041 (2014).

[25] J. L. Burchnall and T. W. Chaundy, Commutative ordinary differential operators, Proc. London Math. Soc. Ser 2 21, 420 (1923); Commutative ordinary differential operators, Proc. R. Soc. A 118, 557 (1928).

[26] A. R. Its and V. B. Matveev, Schrödinger operators with finitegap spectrum and N-soliton solutions of the Korteweg-de Vries equation, Theor. Math. Phys. 23, 343 (1975).

[27] F. Gesztesy and H. Holden, Soliton Equations and Their Algebro-Geometric Solutions (Cambridge University Press, Cambridge, UK, 2003).

[28] E. D. Belokolos et al., Algebro-Geometric Approach to Nonlinear Integrable Equations (Springer, Berlin, 1994).

[29] M. S. Plyushchay, A. Arancibia, and L.-M. Nieto, Exotic supersymmetry of the kink-antikink crystal, and the infinite period limit, Phys. Rev. D 83, 065025 (2011).

[30] M. J. Ablowitz, D. J. Kaup, A. C. Newell, and H. Segur, Method for Solving the Sine-Gordon Equation, Phys. Rev. Lett. 30, 1262 (1973); The inverse scattering transformfourier analysis for nonlinear problems, Stud. Appl. Math. 53, 249 (1974).

[31] V.E. Zakharov and A. B. Shabat, Exact theory of two-dimensional self-focusing and one-dimensional selfmodulation of waves in nonlinear media, Zh. Eksp. Teor. Fiz. 61, 118 (1971) [Sov. Phys. JETP 34, 62 (1972)].

[32] A. Arancibia, J. M. Guilarte, and M. S. Plyushchay, Fermion in a multi-kink-antikink soliton background, and exotic supersymmetry, Phys. Rev. D 88, 085034 (2013).

[33] I. Kay and H. E. Moses, Reflectionless transmission through dielectrics and scattering potentials, J. Appl. Phys. 27, 1503 (1956). 